\title{
Effects of outflow from the surface of a sphere on drag, shear lift, and scalar diffusion
}

\section{$\operatorname{AUTHOR}(\mathrm{S})$ :}

Kurose, R; Makino, H; Komori, S; Nakamura, M; Akamatsu, F; Katsuki, M

\section{CITATION:}

Kurose, R ... [et al]. Effects of outflow from the surface of a sphere on drag, shear lift, and scalar diffusion. PHYSICS OF FLUIDS 2003, 15(8): 2338-2351

\section{ISSUE DATE:}

2003-08

URL:

http://hdl.handle.net/2433/39783

\section{RIGHT:}

Copyright 2003 American Institute of Physics. This article may be downloaded for personal use only. Any other use requires prior permission of the author and the American Institute of Physics. 


\title{
Effects of outflow from the surface of a sphere on drag, shear lift, and scalar diffusion
}

\author{
Ryoichi Kurose ${ }^{\text {a) }}$ and Hisao Makino \\ Yokosuka Research Laboratory, Central Research Institute of Electric Power Industry (CRIEPI), \\ 2-6-1 Nagasaka, Yokosuka 240-0196, Japan \\ Satoru Komori \\ Department of Mechanical Engineering, Kyoto University, Kyoto 606-8501, Japan
}

Mariko Nakamura, Fumiteru Akamatsu, and Masashi Katsuki

Department of Mechanical Engineering, Osaka University, Osaka 565-0871, Japan

(Received 5 July 2002; accepted 22 May 2003; published 2 July 2003)

\begin{abstract}
A three-dimensional numerical simulation of an isothermal flow past a solid sphere with outflow in a linear shear flow is performed to investigate the effects of the outflow on drag and shear lift. In addition, the effects of the outflow and the fluid shear on diffusion and reaction of reactant from the surface of the sphere are also discussed. The results show that the outflow reduces the drag, and, in the linear shear flow, acts to push the sphere to the lower fluid velocity side and promote the negative lift for the high particle Reynolds numbers. The diffusion and reaction of the reactant from the surface of the sphere are strongly affected by the outflow and the fluid shear because these factors cause the deformation of vortices appearing behind the sphere. () 2003 American Institute of Physics. [DOI: 10.1063/1.1591770]
\end{abstract}

\section{INTRODUCTION}

Since the dispersion of droplets or solid particles in air flows is seen in a number of industrial processes and environments, understanding their behaviors is of great importance in designing industrial equipment and resolving environmental problems.

The motion of a droplet or solid particle in an air flow is mainly dominated by drag, and hence approximate expressions for the drag acting on a sphere have been proposed as a function of the particle Reynolds number. ${ }^{1,2}$ However, when considering the motion of a fuel droplet or fuel solid particle, which is utilized in energy conversion devices such as gas turbine engines and pulverized coal combustion furnaces, the effect of outflow from the surface of the fuel droplet or solid particle due to evaporation on the drag should be taken into account. ${ }^{3-8}$ It is well known that the outflow tends to reduce the drag. Renksizbulut and Yuen ${ }^{5}$ performed numerical simulations of the flow past an evaporating droplet under the assumption that the flow field is axisymmetrical and steady without turbulence and viscous dissipation, and showed that the outflow reduces friction drag, but increases pressure drag. On the other hand, when the droplet or solid particle is moving in a shear flow, a transverse force referred to as shear lift is exerted. The shear lift acting on a sphere in a linear shear flow has often been investigated, and some analytical or approximate expressions for it have been proposed as functions of particle Reynolds number and fluid shear rate. ${ }^{9-12}$ However, as far as we know, no one has shown the effect of the outflow on the shear lift.

The heat and mass transfer from a droplet or a solid

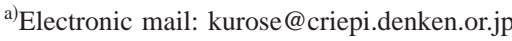

particle has also been studied by numbers of researchers using two analogous dimensionless parameters for the heat and mass transfer rates; those are Nusselt number $(\mathrm{Nu})$ and Sherwood number (Sh). Dandy and Dwyer, ${ }^{13}$ Tieng and Yan, ${ }^{14}$ Nguyen et al., ${ }^{15}$ Chattopadhyay and Dash, ${ }^{16}$ Kawahara et al. ${ }^{17}$ Balachandar and $\mathrm{Ha},{ }^{18}$ and Blackburn ${ }^{19}$ investigated the effects of fluid shear, particle spin, gravity, radial mass efflux, acoustic streaming, unsteadiness, and oscillatory flows on the heat and mass transfer rates, respectively. Regarding the effect of the fluid shear, Dandy and Dwyer, ${ }^{13}$ who performed a three-dimensional numerical simulation of a linear shear flow around a heated solid sphere, stated that $\mathrm{Nu}$ averaged on the surface of the solid sphere is essentially independent of the fluid shear rate. However, Misumi et al. ${ }^{20}$ and Komori and Misumi ${ }^{21}$ recently did a similar numerical simulation of a linear shear flow around a bubble with a slip surface boundary, and showed that although Sh averaged on the bubble is not affected by the fluid shear, the values of Sh on the local places, referred as to local Sherwood number, $\mathrm{Sh}_{\text {local }}$, are clearly different.

In the present study, a three-dimensional numerical simulation of an isothermal flow past a solid sphere with outflow in a linear shear flow is performed to investigate the effects of the outflow on the drag and shear lift. In addition, the effects of the outflow and fluid shear on the diffusion and reaction of reactants introduced from the surface of the sphere and upstream are also discussed.

\section{NUMERICAL SIMULATION}

The flow geometry, coordinate system and computational domain for the computations of the isothermal flow around a sphere are shown in Figs. 1 and 2. The imposed 


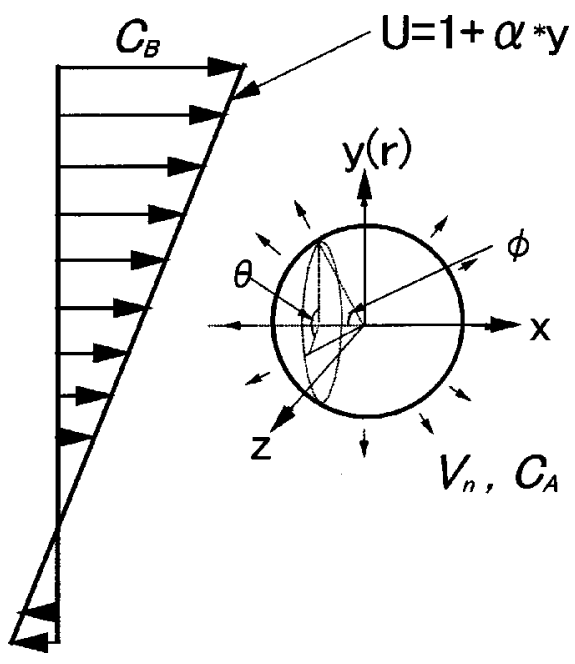

FIG. 1. Coordinate system for a linear shear flow around a sphere with outflow from the surface.

flow is a linear shear flow around a sphere with outflow from the surface. The three-dimensional unsteady Navier-Stokes $(\mathrm{N}-\mathrm{S})$ equations in cylindrical coordinates are given by

$\frac{\partial U}{\partial t}+(\mathbf{V} \cdot \nabla) U=-\frac{\partial p}{\partial x}+\frac{2}{\operatorname{Re}} \nabla^{2} U$,

$\frac{\partial V}{\partial t}+(\mathbf{V} \cdot \boldsymbol{\nabla}) V-\frac{V^{2}}{r}=-\frac{\partial p}{\partial r}+\frac{2}{\operatorname{Re}}\left(\nabla^{2} V-\frac{V}{r^{2}}-\frac{2}{r^{2}} \frac{\partial V}{\partial \theta}\right)$,

$$
\begin{aligned}
\frac{\partial W}{\partial t} & +(\mathbf{V} \cdot \nabla) W+\frac{V W}{r} \\
& =-\frac{1}{r} \frac{\partial p}{\partial \theta}+\frac{2}{\operatorname{Re}}\left(\nabla^{2} W-\frac{W}{r^{2}}+\frac{2}{r^{2}} \frac{\partial W}{\partial \theta}\right) .
\end{aligned}
$$

Here, Re is the particle Reynolds number, defined by

$$
\operatorname{Re}=\frac{2 a U_{c}}{\nu},
$$

where $a$ is the radius of the sphere, $\nu$ is the kinematic viscosity, and $U_{c}$ is the mean velocity of the fluid on the streamline through the center of the sphere. The $\mathrm{N}-\mathrm{S}$ equations are directly solved using a finite difference scheme based on the

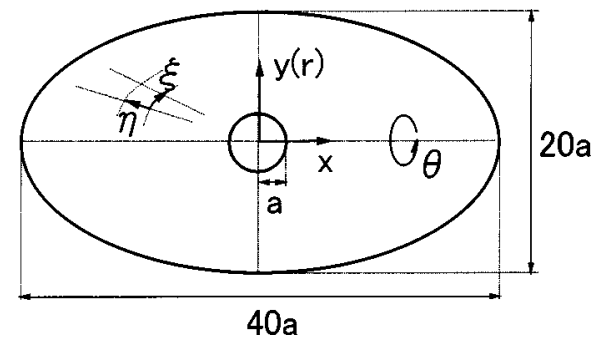

FIG. 2. Schematic of computational domain for a linear shear flow around a sphere.

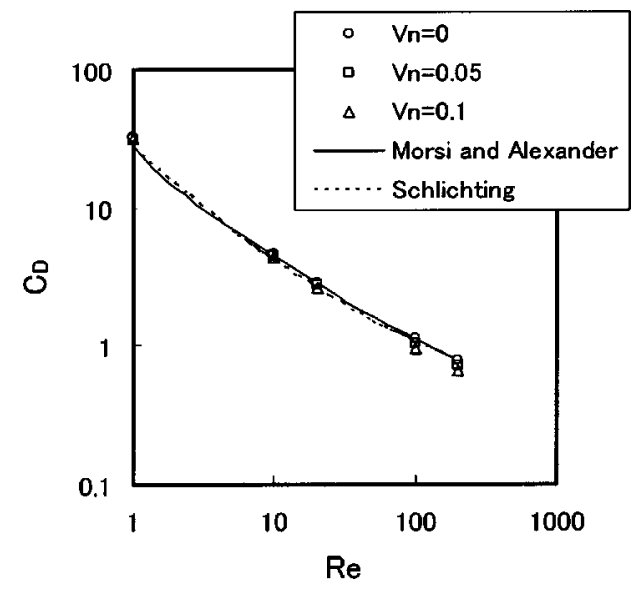

FIG. 3. Effect of outflow velocity, $V_{n}$, on drag coefficient, $C_{D}\left(\alpha^{*}=0\right)$.

marker and cell (MAC) method. By taking divergence of the $\mathrm{N}-\mathrm{S}$ equations, the dimensionless pressure-Poisson (p-P) equation is derived as follows:

$$
\nabla^{2} p=\frac{\partial D}{\partial t}-\nabla \cdot[(\mathbf{V} \cdot \nabla) \mathbf{V}]+\frac{2}{\operatorname{Re}} \nabla^{2} D .
$$

The $\mathrm{N}-\mathrm{S}$ equations and the p-P equation are solved alternately. Here, $D, \mathbf{V}$, and $\nabla^{2}$ are defined by

$$
\begin{aligned}
& D=\boldsymbol{\nabla} \cdot \mathbf{V}, \\
& \mathbf{V}=\left(V_{x}, V_{r}, V_{\theta}\right)=(U, V, W), \\
& \nabla^{2}=\frac{\partial^{2}}{\partial x}+\frac{1}{r} \frac{\partial}{\partial r}\left(r \frac{\partial}{\partial r}\right)+\frac{1}{r^{2}} \frac{\partial^{2}}{\partial \theta^{2}} .
\end{aligned}
$$

The numerical procedure used here is essentially the same as used in Hanazaki ${ }^{22}$ and Kurose and Komori. ${ }^{12}$ The boundary condition on the surface of the sphere is given by a nonslip or an outflow condition. For the outflow condition, the outflow velocity, $V_{n}$, is defined as

$$
\mathbf{V} \cdot \mathbf{n}=V_{n}, \quad \text { for } r=a,
$$

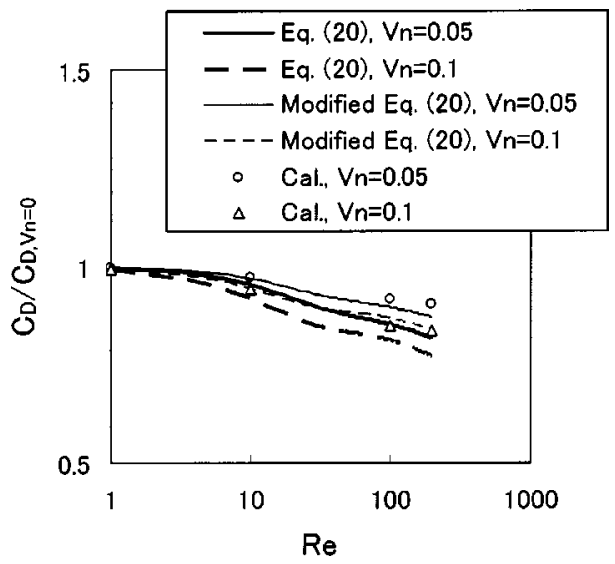

FIG. 4. A comparison of the ratio of the drag coefficient with outflow velocity, $C_{D}$, to that without outflow velocity, $C_{D, V_{n=0}}$. 
(a)
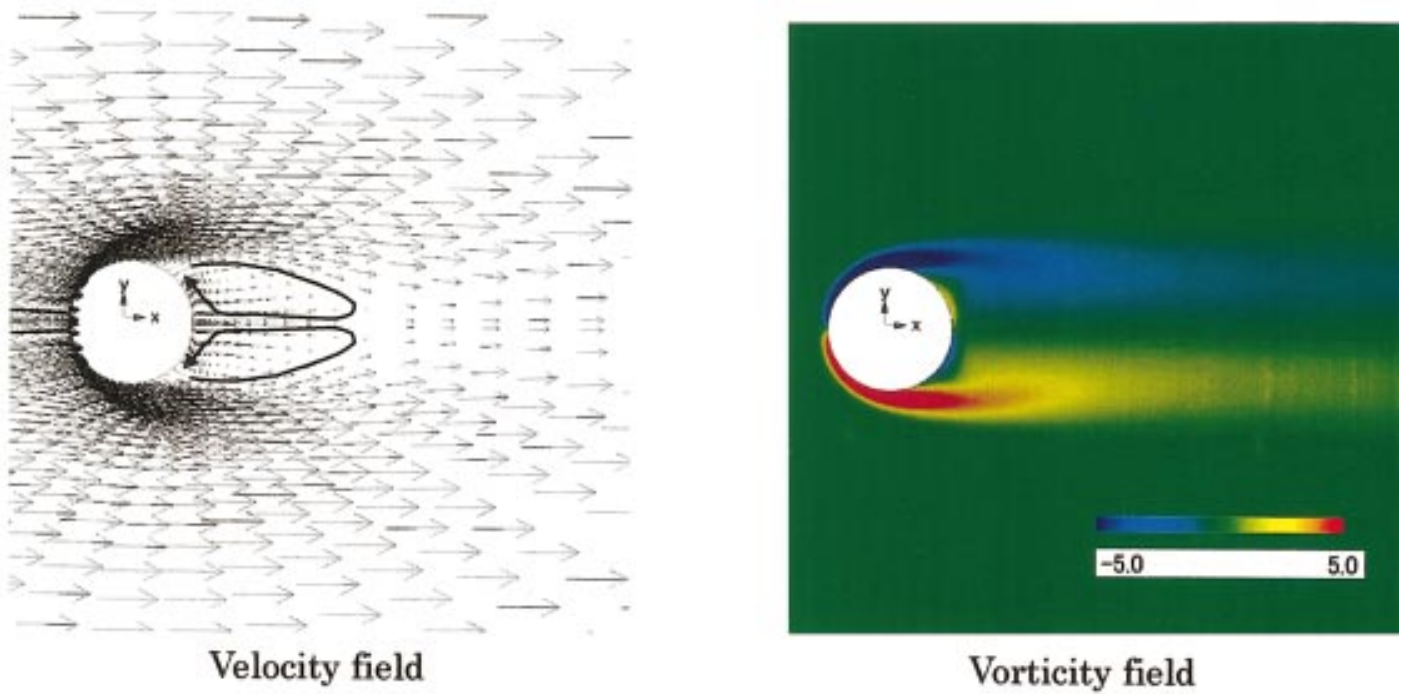

Vorticity field

(b)

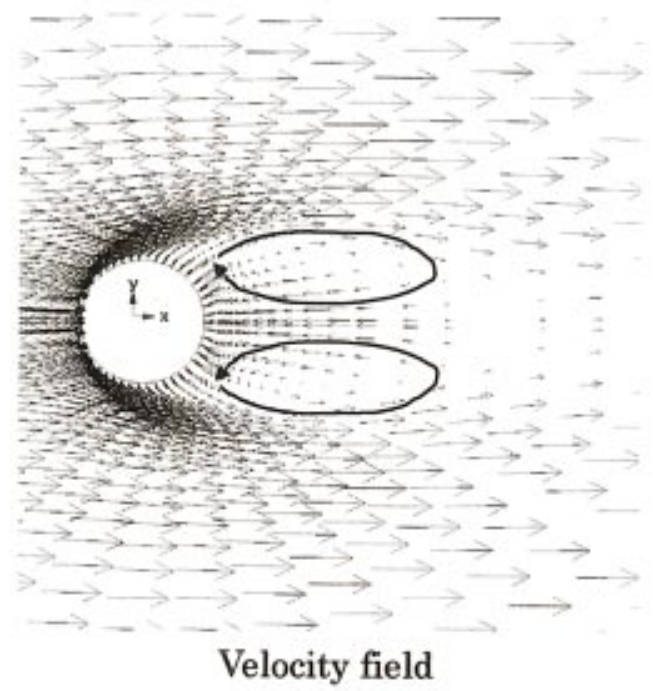

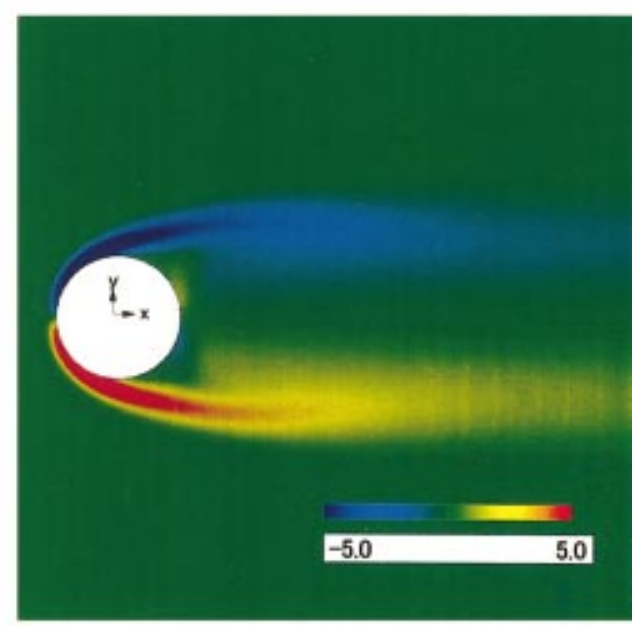

Vorticity field

FIG. 5. (Color) Velocity and vorticity fields around a sphere in a uniform unsheared flow $\left(\operatorname{Re}=200, \alpha^{*}=0\right)$ : (a) $V_{n}=0$; (b) $V_{n}=0.1$.

where $\mathbf{n}$ is the outward unit normal to the surface of the sphere, and $V_{n}$ is nondimensionalized using $U_{c}$. The boundary condition of velocity upstream of a sphere is given in a dimensionless form by

$$
U=1+\alpha^{*} y,
$$

where $\alpha^{*}$ is the dimensionless shear rate of fluid defined by

$$
\alpha^{*}=\left(\frac{a}{U_{c}}\right)\left(\frac{\partial U}{\partial y}\right) .
$$

The velocity condition on the outer boundary, except upstream, is

$$
\frac{\partial \mathbf{V}}{\partial x}=0 .
$$

When considering the one-step irreversible, secondorder chemical reaction between chemical species $A$ and $B$, which are introduced from the surface of the sphere and upstream, respectively (Fig. 1), in an isothermal flow, $A+B$ $\rightarrow P$, the conservation equations can be written for the concentration $C$ of $A, B$, and $P$ as follows:

$$
\begin{aligned}
& \frac{\partial C_{A}}{\partial t}+(\mathbf{V} \cdot \nabla) C_{A}=\frac{2}{\operatorname{ReSc}} \nabla^{2} C_{A}-\mathrm{Da} C_{A} C_{B}, \\
& \frac{\partial C_{B}}{\partial t}+(\mathbf{V} \cdot \nabla) C_{B}=\frac{2}{\operatorname{ReSc}} \nabla^{2} C_{B}-\mathrm{Da} C_{A} C_{B}, \\
& \frac{\partial C_{P}}{\partial t}+(\mathbf{V} \cdot \nabla) C_{P}=\frac{2}{\operatorname{ReSc}} \nabla^{2} C_{P}+\operatorname{Da} C_{A} C_{B},
\end{aligned}
$$




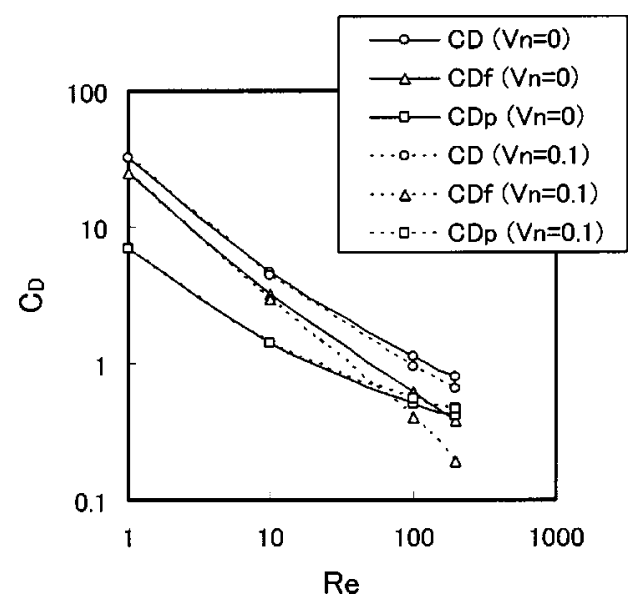

FIG. 6. Contributions of friction, $C_{D f}$, and pressure, $C_{D p}$, to the drag coefficient, $C_{D}$, for $V_{n}=0$ and $0.1\left(\alpha^{*}=0\right)$.

(a)

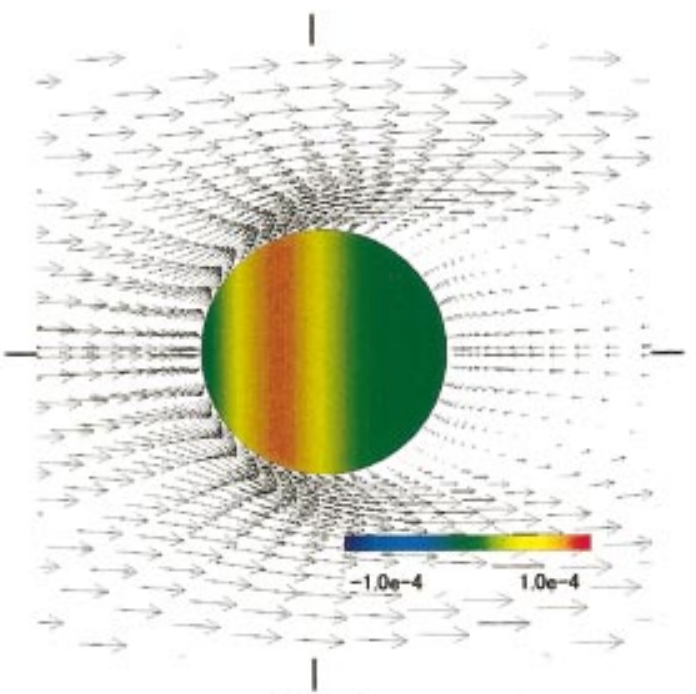

Friction

(b)

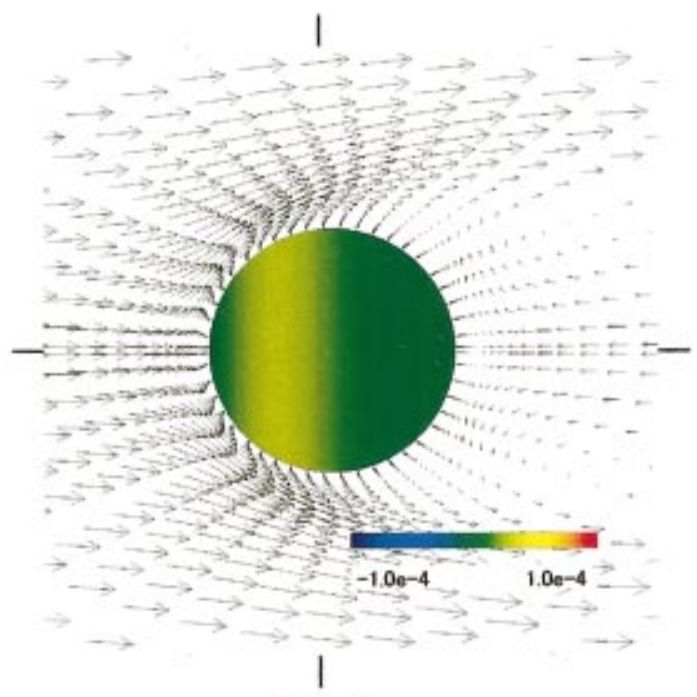

Friction where Sc and Da are the Schmidt number and the Damköhler number, defined by

$$
\mathrm{Sc}=\frac{\nu}{D}, \quad \mathrm{Da}=\frac{k a C_{A 0}}{U_{c}}
$$

where $D$ and $k$ are the diffusivity and the reaction rate constant, which are assumed to be identical for all species.

By using a grid generation method developed by Thames et al. ${ }^{23}$ grid points are concentrated near the surface of the sphere in the $(x, r)$ plane. The maximum sizes of the computational domain are 20 and 10 radii in the $x$ and $r$ directions, respectively. The $(x, r, \theta)$-coordinate system is transformed to the $(\eta, \zeta, \theta)$-coordinate system with an equal grid spacing (Fig. 2). The grid points used in this study are 70 $\times 120 \times 96$ in the $\eta, \zeta$, and $\theta(0 \leqslant \theta \leqslant 2 \pi)$ directions. The

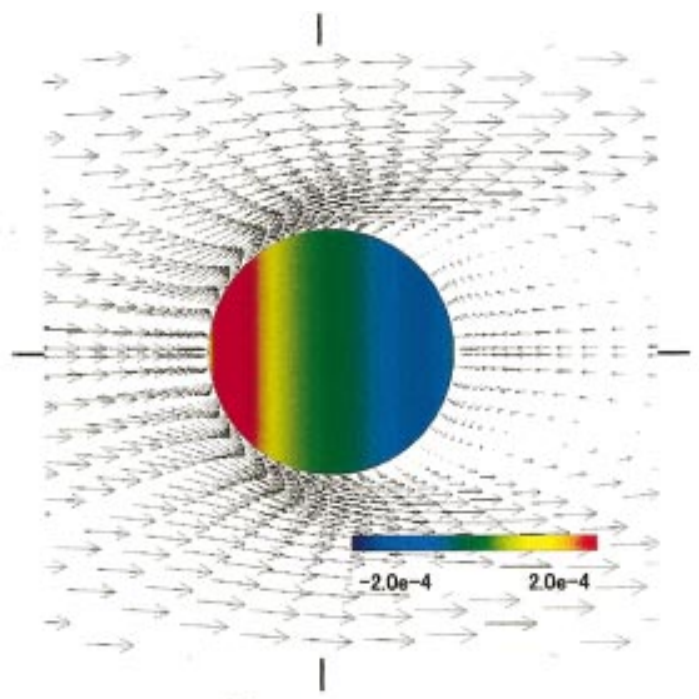

Pressure

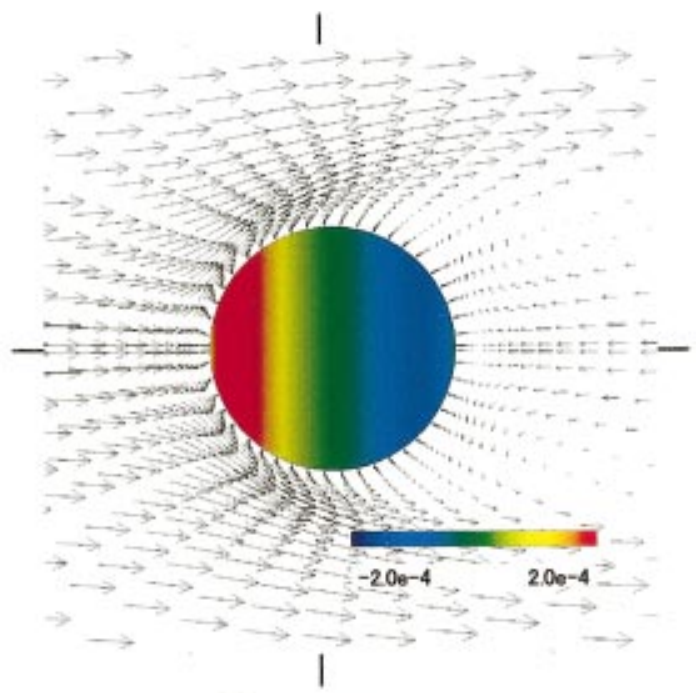

Pressure

FIG. 7. (Color) Surface contours of the $x$-component of instantaneous friction, $\tau$, and pressure, $p\left(\operatorname{Re}=200, \alpha^{*}=0\right)$ : (a) $V_{n}=0$; (b) $V_{n}=0.1$. 


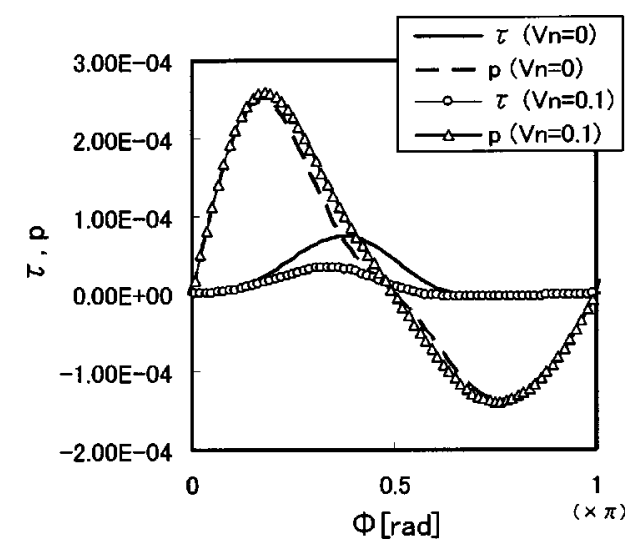

FIG. 8. Surface friction, $\tau$, and pressure, $p$, distributions at $\theta=0$ against $\phi$ for $V_{n}=0$ and $0.1\left(\operatorname{Re}=200, \alpha^{*}=0\right)$.

(a)

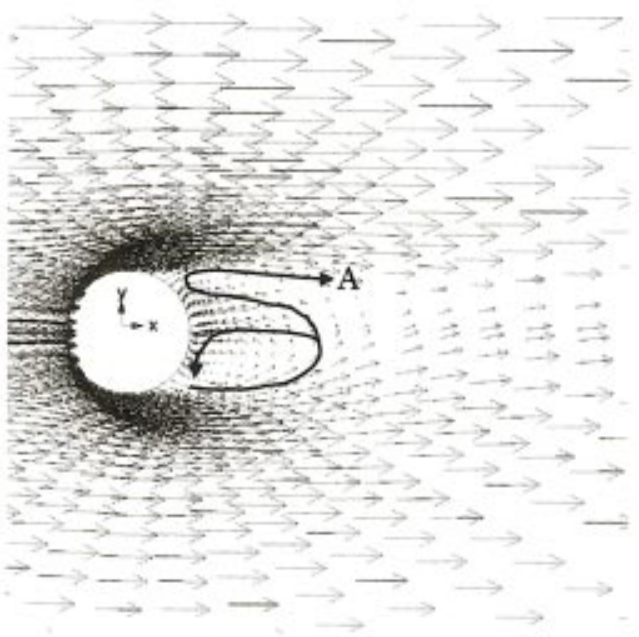

Velocity field

(b)

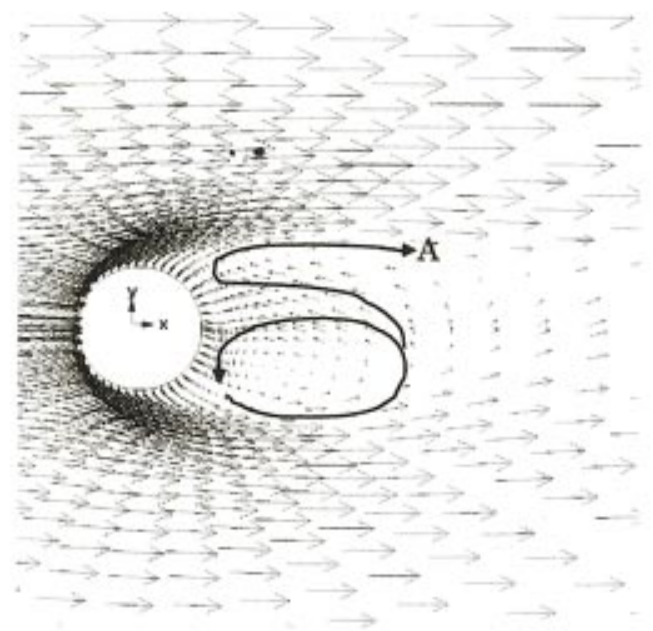

Velocity field transformed governing equations are discretized to construct the finite difference formulation. The nonlinear terms in the $\mathrm{N}-\mathrm{S}$ equations are approximated by a third-order scheme of Kawamura and Kuwahara, ${ }^{24}$ and the other spatial derivatives are approximated by a second-order central difference scheme. The dimensionless time step $\Delta t$ is 0.01 .

The drag and lift, $F_{D}$ and $F_{L}$, are the components of the fluid force acting on the sphere in the $x$ and $y$ directions, and they can be calculated by the sum of the contributions of pressure and friction:

$$
F_{i}=F_{i, p}+F_{i, f}=-\int_{S} p \mathbf{e}_{i} \cdot \mathbf{n} \cdot d S+\int_{S} \mathbf{n} \cdot \boldsymbol{\tau} \cdot \mathbf{e}_{i} d S,
$$

where $\mathbf{n}$ is the outward unit normal to the surface of the
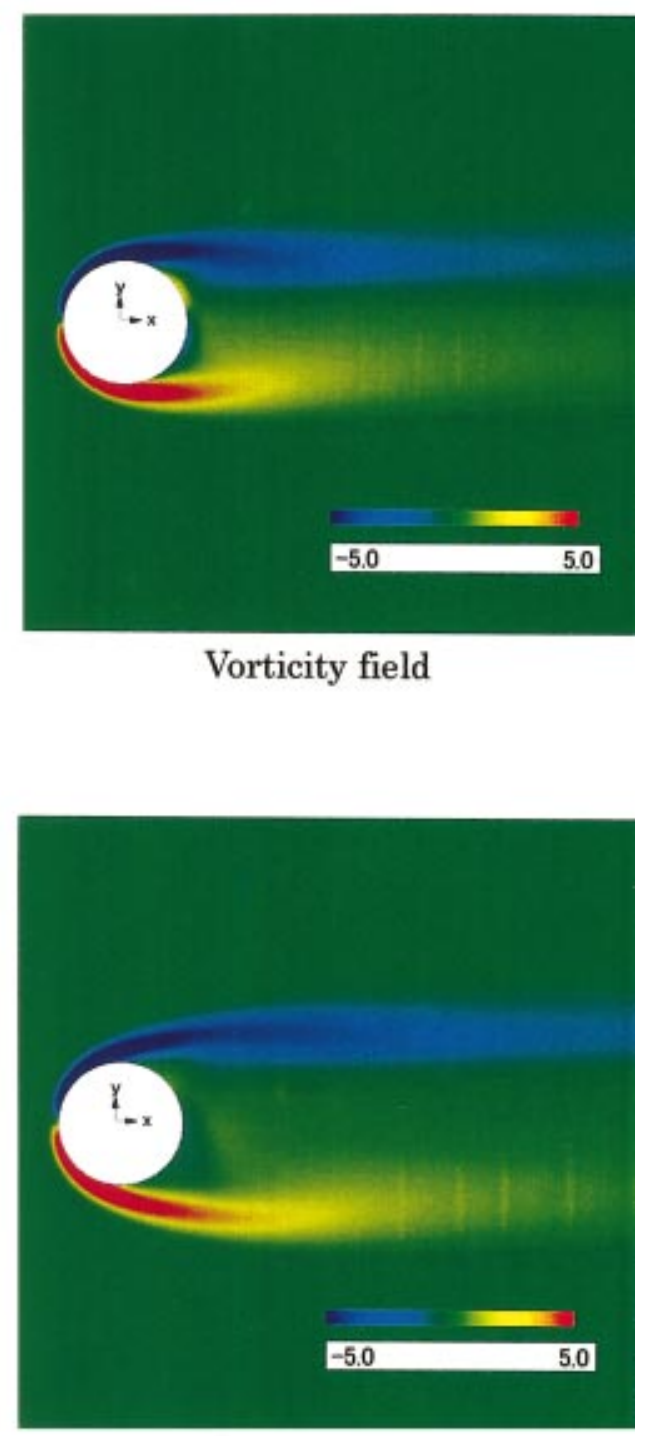

Vorticity field

FIG. 9. (Color) Velocity and vorticity fields around a sphere in a linear shear flow $\left(\operatorname{Re}=200, \alpha^{*}=0.1\right)$ : (a) $V_{n}=0 ;(\mathrm{b}) V_{n}=0.1$. 


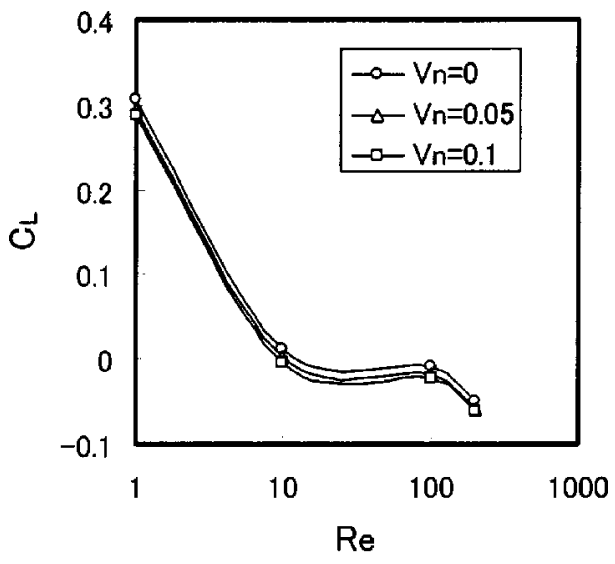

FIG. 10. Effect of outflow velocity, $V_{n}$, on lift coefficient, $C_{L}\left(\alpha^{*}=0.1\right)$.

sphere and $\boldsymbol{\tau}$ is the viscous stress tensor. The drag and lift coefficients, $C_{D}$ and $C_{L}$, are defined by

$$
C_{i}=\frac{F_{i}}{\frac{1}{2} \rho_{f} U_{c}^{2} \pi a^{2}},
$$

where $\rho_{f}$ is the fluid density.

The dimensionless mass transfer rate from the sphere is obtained as the Sherwood number, Sh, by integrating the concentration gradient of the reactant $A$ :

$$
\mathrm{Sh}=-\left.\frac{1}{2 \pi a} \int_{S} \frac{\partial C_{A}}{\partial \eta}\right|_{\eta=1} d S .
$$

The computations were performed for the particle Reynolds numbers of $\operatorname{Re}=1,10,20,100,200$, the outflow velocities of $V_{n}=0,0.05,0.1$, and the fluid shear rates of $\alpha^{*}=0,0.1$. For the calculations of the species concentrations, the Schmidt number, Sc, was set at 1.0 , and the Damköhler number, Da, was 0 or 0.1 . Simulations were run 10000 time steps, and the CPU time required for each case was $3.0 \mathrm{~h}$ on a Fujitsu VPP5000 supercomputer.

\section{RESULTS AND DISCUSSION}

In developing new technologies for the energy conversion devices, understanding the behavior of the fuel particles especially in the region near the burner is essential. In this region, it is speculated that there exists a strong fluid shear due to the jet and swirl, and the slip velocity, which is the velocity difference between the fluid and particle, is up to be of $10^{1}$ order of magnitude $(\mathrm{m} / \mathrm{s})$. On the other hand, the maximum dimensional outflow velocity for a single droplet in a flame is estimated to be of $10^{\circ}$ order of magnitude $(\mathrm{m} / \mathrm{s})$ (see the Appendix). Hence, it would be important to understand the behaviors of the drag, lift, and scalar diffusion for the evaporating fuel particles, with the dimensionless outflow velocity of up to $V_{n}=O(-1)$ in details. Here, $V_{n}$ is nondimensionalized using the slip velocity. However, unfortunately, since the converged result for the larger outflow velocity of $V_{n}>0.1$ under the existence of the fluid shear

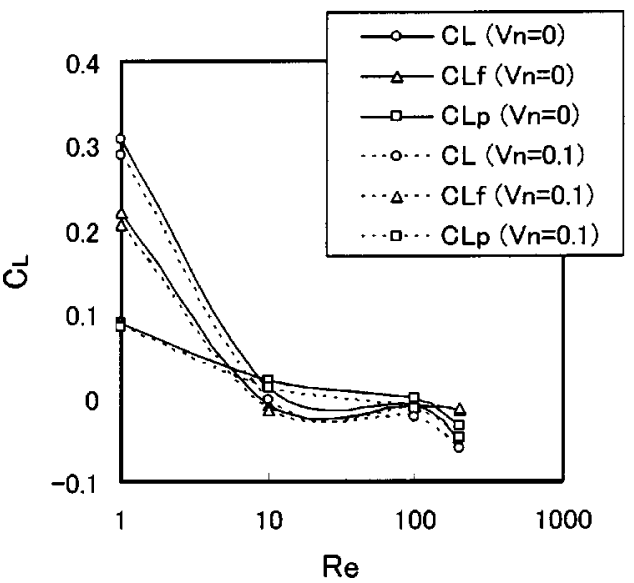

FIG. 11. Contributions of friction, $C_{L f}$, and pressure, $C_{L p}$, to lift coefficient, $C_{L}$, for $V_{n}=0$ and $0.1\left(\alpha^{*}=0.1\right)$.

could not be obtained using our numerical code, the effect of the outflow velocity in the region of $0 \leqslant V_{n} \leqslant 0.1$ is provided here.

\section{A. Effect of outflow on drag}

Figure 3 shows the effect of the outflow velocity, $V_{n}$, on the drag coefficient, $C_{D}$, acting on a sphere in a uniform unsheared flow $\left(\alpha^{*}=0\right)$ together with the previous experiments in Schlichting ${ }^{25}$ and the approximate expressions of Morsi and Alexander. ${ }^{2}$ The present $C_{D}$ for $V_{n}=0$ is in good agreement with the experiments ${ }^{25}$ and the approximate expressions $^{2}$ for $V_{n}=0$. The value of $C_{D}$ decreases with increasing $V_{n}$ for a fixed value of Re. The dependence of $C_{D}$ on $V_{n}$ is more obvious for higher Re and the difference in $C_{D}$ between $V_{n}=0$ and 0.1 exceeds $16 \%$ at $\mathrm{Re}=200$. The reduction of $C_{D}$ due to the outflow is a well-known phenomenon, and the behavior has been investigated over the past 50 years. The ratio of the drag coefficient with the outflow velocity, $C_{D}$, to that without the outflow velocity, $C_{D, V_{n=0}}$, is shown in Fig. 4, together with the empirical expression generally used: ${ }^{26,27}$

$$
\begin{aligned}
& C_{D}=\frac{24}{\operatorname{Re}}\left(\frac{1+0.545 \mathrm{Re}+0.1 \mathrm{Re}^{1 / 2}(1-0.03 \mathrm{Re})}{1+a\left|\operatorname{Re}_{n}\right|^{b}}\right), \\
& a=0.09+0.077 \exp (-0.4 \mathrm{Re}), \\
& b=0.4+0.77 \exp (-0.04 \mathrm{Re}),
\end{aligned}
$$

where $\operatorname{Re}_{n}=2 a V_{n}^{*} / \nu$. Here, $V_{n}^{*}$ is the dimensional outflow velocity. Although both $C_{D} / C_{D, V_{n=0}}$ decrease with increasing $\mathrm{Re}$ and be of general agreement with each other for $\operatorname{Re} \leqslant 10$, the empirical expression tends to overestimate the effect of the outflow for larger Re. This expression tends to approach the numerical values by modifying the parameter $a$ as

$a=0.06+0.077 \exp (-0.4 \mathrm{Re})$.

Figure 5 shows the velocity and vorticity fields around the sphere with and without the outflow velocities of $V_{n}$ $=0$ and 0.1 for the particle Reynolds number of $R e=200$ on 
(a)

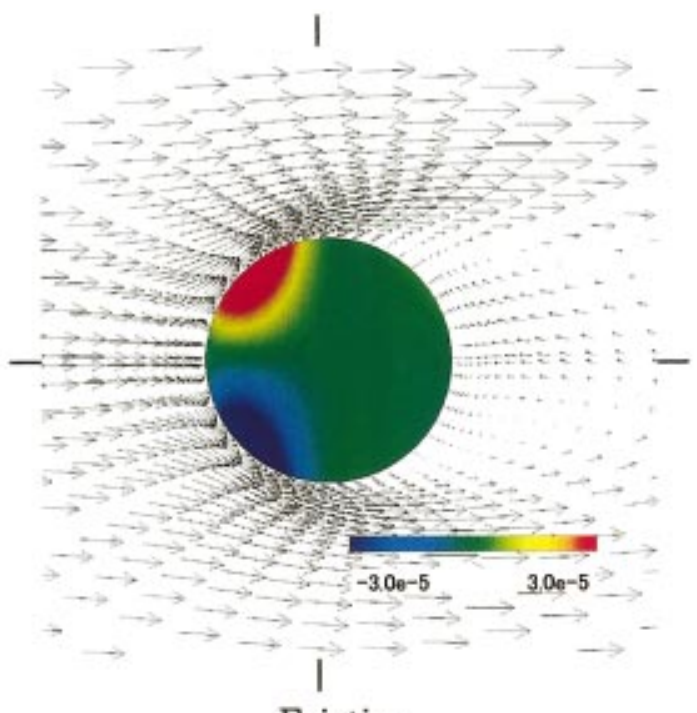

Friction

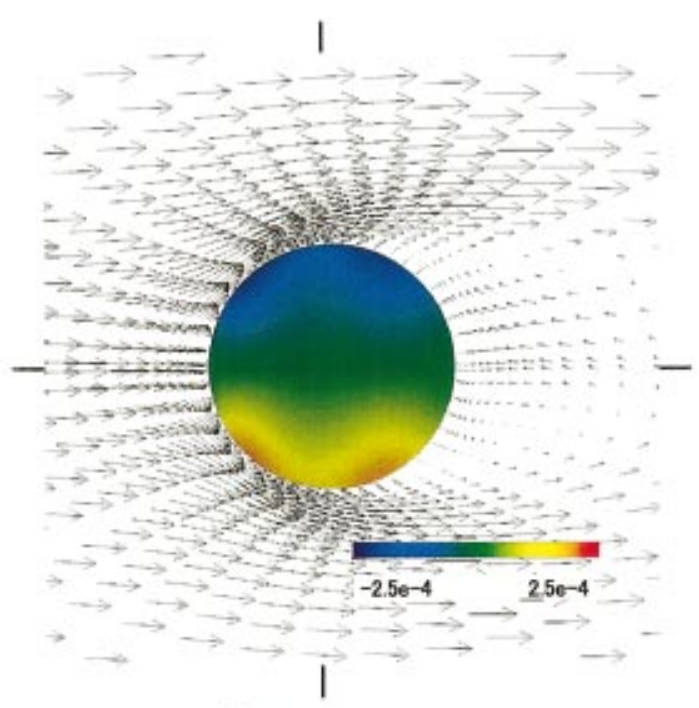

Pressure (b)

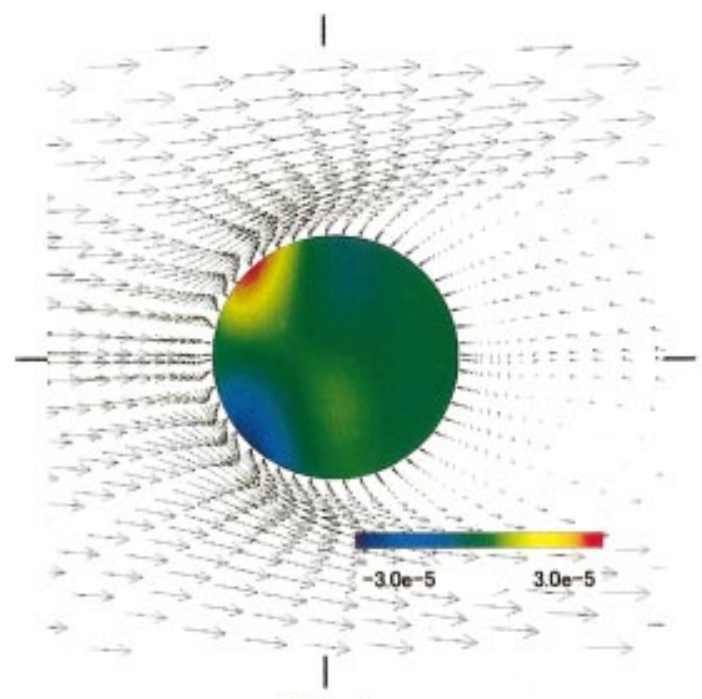

Friction

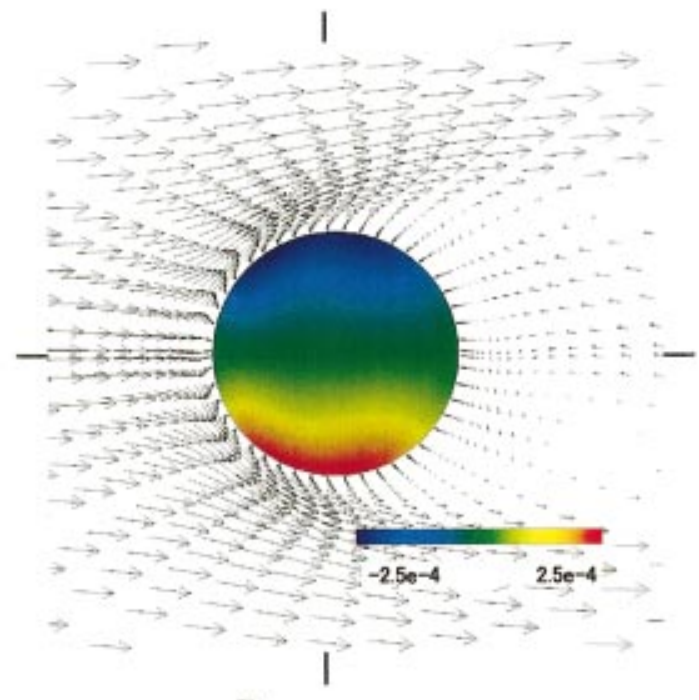

Pressure

FIG. 12. (Color) Surface contours of the $y$-component of instantaneous friction, $\tau$, and pressure, $p\left(\operatorname{Re}=200, \alpha^{*}=0.1\right)$ : (a) $V_{n}=0$; (b) $V_{n}=0.1$.

the centerplane $(z=0)$. For both outflow conditions, symmetry with regard to the $x$ axis is observed. Compared to the outflow velocity of $V_{n}=0$, the vortices generated behind the sphere with $V_{n}=0.1$ move downward and the sizes become large. Also, it is found that, for $V_{n}=0.1$, the strong vorticity region expands in the radial direction and the points of the flow separation, where tangential viscous stress is zero on the surface, shift upward slightly.

Renksizbulut and Yuen ${ }^{5}$ performed numerical simulations of the flow past an evaporating droplet under the assumption that the flow field is axisymmetrical and steady without turbulence and viscous dissipation, and showed that the outflow reduces friction drag, but increases pressure drag. To verify the effects of the outflow on the friction and pressure drags, the contributions of the friction, $C_{D f}$, and pressure, $C_{D p}$, to the total drag coefficient, $C_{D}$, for $V_{n}=0$ and
0.1 are shown in Fig. 6. As stated by Renksizbulut and Yuen, ${ }^{5}$ the reduction of $C_{D f}$ and the increase of $C_{D p}$ due to the outflow are observed. It is also found that the reduction of the total drag, $C_{D}$, is caused by the fact that the rate of reduction of $C_{D f}$ is greater than the rate of increase of $C_{D p}$. These behaviors of $C_{D f}$ and $C_{D p}$ are confirmed in Figs. 7 and 8 , which show the surface contours of the $x$ component of the instantaneous friction, $\tau$, and pressure, $p$, and their surface distributions at $\theta=0$ against $\phi$ for $V_{n}=0$ and 0.1 $(\operatorname{Re}=200)$. For the outflow velocity of $V_{n}=0.1$, the high friction located on the surface just upstream at $\phi=\pi / 2$ appeared for the outflow velocity of $V_{n}=0$ is clearly weakened. This reduction of $\tau$ appeared on the top and bottom of the sphere is considered to be associated with the boundary layer thickness. The boundary layer thickness on the sphere with the outflow becomes thicker than that without the outflow espe- 
(a)

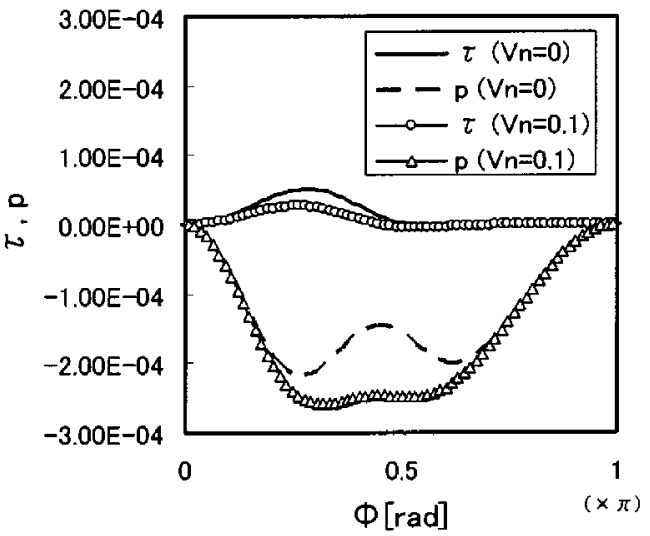

(b)

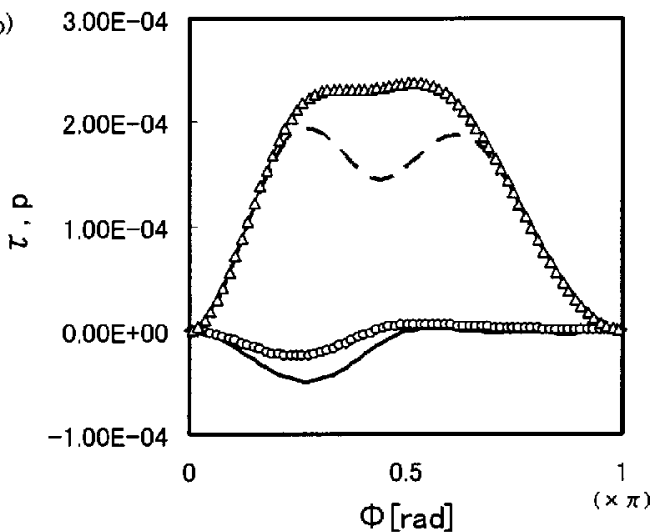

(c)

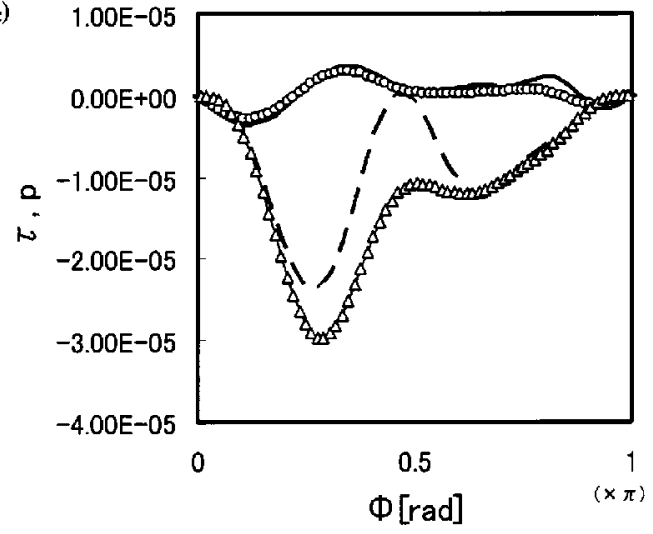

FIG. 13. Surface friction, $\tau$, and pressure, $p$, distributions against $\phi$ for $V_{n}$ $=0$ and $0.1\left(\operatorname{Re}=200, \alpha^{*}=0.1\right)$ : (a) top $(\theta=0)$; (b) bottom $(\theta=\pi)$; (c) sum of the top and bottom.

cially on the top and bottom (see the velocity fields in Fig. 7), which reduces the friction in the boundary layer. On the other hand, these pressure distributions are slightly different on the surface in the region of $\pi / 4 \leqslant \phi \leqslant 3 \pi / 4$.

\section{B. Effect of outflow on shear lift}

Figure 9 shows the velocity and vorticity fields around a sphere with and without the outflow velocities of $V_{n}=0$ and 0.1 in a linear shear flow $\left(\alpha^{*}=0.1\right)$ for the particle Reynolds number of $\operatorname{Re}=200$ on the centerplane $(z=0)$. As described in Kurose and Komori, ${ }^{12}$ the vortices generated behind the sphere are found to be deformed by the fluid shear. The upper (higher fluid velocity side) vortex is flattened and nearly broken by the influent from the lower (lower fluid velocity side) vortex (arrow $A$ in Fig. 9). It is also observed that the strong vorticity region on the upper side stretches and that on the lower side shrinks downstream, and that the points of the flow separation slightly upward and downward on the upper and lower sides, respectively. Similar to the uniform unsheared flow in Fig. 5, due to the outflow velocity, these vortices move downward and become large. The strong vorticity region for the outflow velocity of $V_{n}=0.1$ expands in the radial direction and the points of the flow separation on both the upper and lower sides shift upward.

Figure 10 shows the effect of the outflow velocity, $V_{n}$, on the lift coefficient, $C_{L}$, acting on the sphere. It is found that $C_{L}$ for all outflow conditions decreases and its sign changes from positive to negative with increasing Re, and that the outflow tends to encourage the negative force and lessen the critical particle Reynolds number. Regarding the negative $C_{L}$ on the sphere without the outflow in a linear shear flow, Kurose and Komori ${ }^{12}$ previously explained the mechanism by introducing the viscous and pressure contributions to the total lift, $C_{L}$. With respect to $C_{D}$ on the sphere in the linear shear flow, it is reported that $C_{D}$ increases with increasing $\alpha^{*}$ for a fixed Re. ${ }^{12,13}$ This trend was also observed for the outflow conditions. Compared to in the uniform unsheared flow $C_{D}$ on the sphere with outflow in the linear shear flow indicated a slightly larger value.

To investigate the effect of $V_{n}$ on $C_{L}$ in detail, the contributions of the friction, $C_{L f}$, and pressure, $C_{L p}$, to the total lift coefficient, $C_{L}$, for the outflow velocities of $V_{n}=0$ and 0.1 are shown in Fig. 11. The trends of $C_{L f}$ and $C_{L p}$ against Re for $V_{n}=0.1$ are similar to those for $V_{n}=0$. As shown in Kurose and Komori, ${ }^{12} C_{L f}$ and $C_{L p}$ decrease with increasing $\mathrm{Re}$, and the tendency is more prominent for $C_{L f}$ in the low particle Reynolds number region of $\operatorname{Re}<10$ and for $C_{L p}$ in the high particle Reynolds number region of $100<\mathrm{Re}$, respectively. Also, when the outflow exists, although both $C_{L f}$ and $C_{L p}$ decrease to promote the negative lift, the effect is stronger for $C_{L f}$ in the low Re region but for $C_{L p}$ in the high $\mathrm{Re}$ region. This behavior is quite different from that of the drag, whose reduction due to the outflow is mainly caused by the decrease in the friction independent of Re (see Fig. 6).

Figures 12 and 13 show the surface contours of the $y$-component of the instantaneous friction, $\tau$, and pressure, $p$, and their surface distributions against $\phi$ for the outflow velocities of $V_{n}=0$ and $0.1(\operatorname{Re}=200)$. In Fig. 13, the distributions of top $(\theta=0)$, bottom $(\theta=\pi)$ and the sum of them are plotted. Due to the outflow, the friction acting on both the top and bottom sides around $\phi=\pi / 4$ weakens, but the pressure acting on the both sides around $\phi=\pi / 2$ strengthens. Totally, the obvious difference appears for the pressure in the region of $\pi / 4 \leqslant \phi \leqslant 3 \pi / 4$, and this pressure difference acts to push the sphere downward.

\section{Effects of outflow and shear lift on reactive scalar diffusion}

Figure 14 shows the effects of the fluid shear and the outflow velocity on the distribution of the reactant $A$ concen- 
(a)

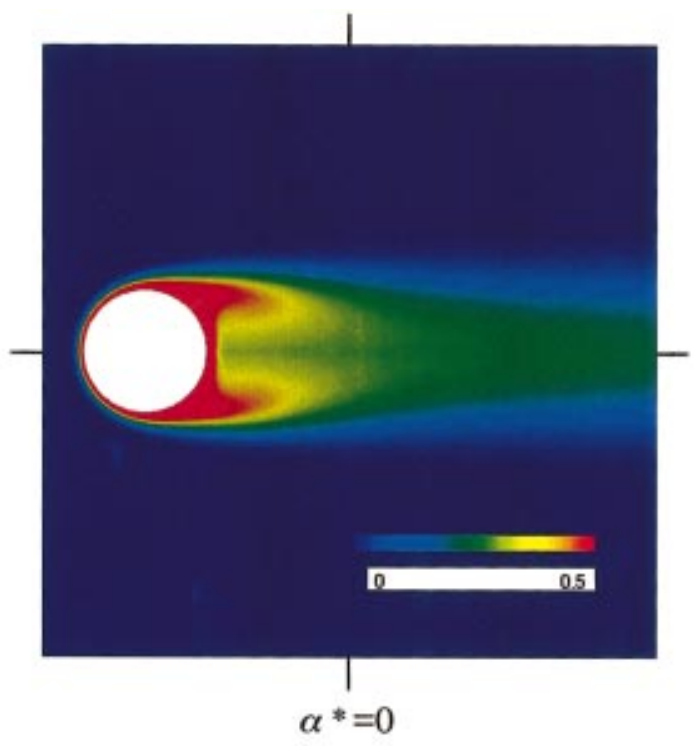

(b)

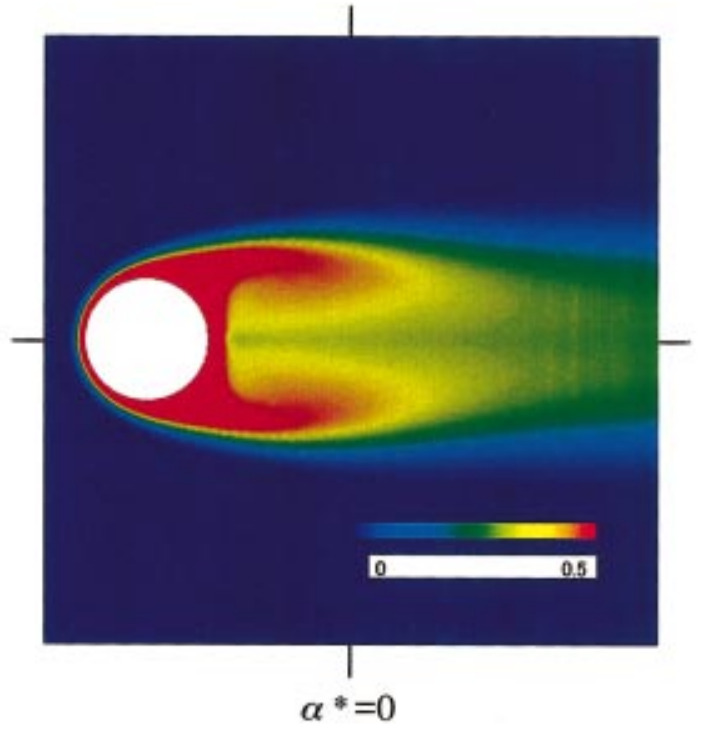

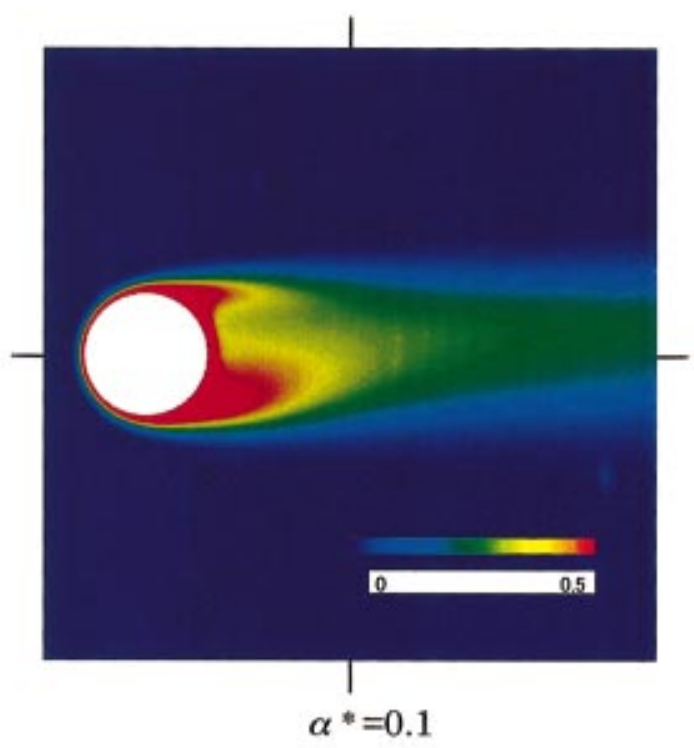

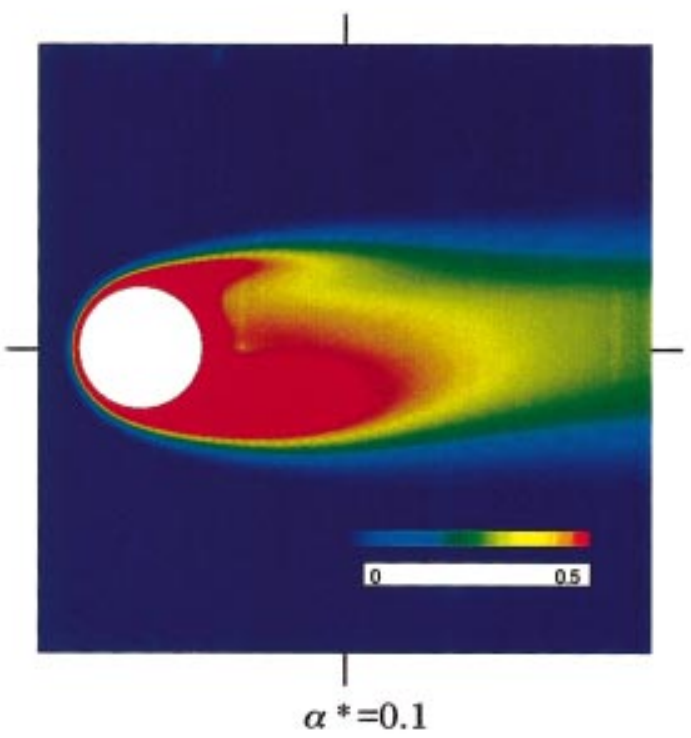

FIG. 14. (Color) Effects of fluid shear rate, $\alpha^{*}$, and outflow velocity, $V_{n}$, on a distribution of reactant concentration, $C_{A}(\operatorname{Re}=200, \mathrm{Da}=0)$ : (a) $V_{n}=0$; (b) $V_{n}=0.1$.

tration, $C_{A}$, for $\mathrm{Re}=200$ and $\mathrm{Da}=0$. As shown in Fig. 1, the reactant $A$ flows out from the surface of the sphere. Although the distributions of $C_{A}$ in uniform unsheared flows $\left(\alpha^{*}=0\right)$ show symmetry with regard to the $x$ axis; those in linear shear flows $\left(\alpha^{*}=0.1\right)$ do not remain symmetrical. It is observed that, in the linear shear flow, a high $C_{A}$ concentration is widely distributed on the lower (lower fluid velocity) side compared to that on the upper (higher fluid velocity) side. Also, the outflow causes the expansion of the high $C_{A}$ concentration area in the radial and downstream directions. The asymmetry of the $C_{A}$ distribution with the $x$ axis for $\alpha^{*}=0.1$ is considered to be associated with the vortices generated behind the sphere. As mentioned before, the upper vortex is flattened and nearly broken by the influent from the lower vortex (arrow $A$ in Fig. 9). A comparison of the $C_{A}$ distribu- tion with the velocity field illustrates that the high $C_{A}$ concentration is on hold in the lower vortex, whereas the high $C_{A}$ concentration area on the upper side is lessened by the breakup of the vortex. Similar effects of the $C_{A}$ distribution in the case of $\mathrm{Da}=0$ are observed for the distribution of the product $P$ concentration, $C_{P}$, in the case of $\mathrm{Da}=0.1$. As shown in Fig. 15, the high $C_{P}$ concentration area is widely distributed on the lower side due to the fluid shear and expanded in the radial and downstream directions due to the outflow. Thus, the diffusion of the reactant from the surface of the sphere and the product is strongly affected by the deformation of the vortices behind the sphere.

To examine the mass transfer rate from the sphere, Fig. 16 shows the effects of the outflow velocity, $V_{n}$, the fluid shear, $\alpha^{*}$, and the Damköhler number, Da, on the Sherwood 
(a)

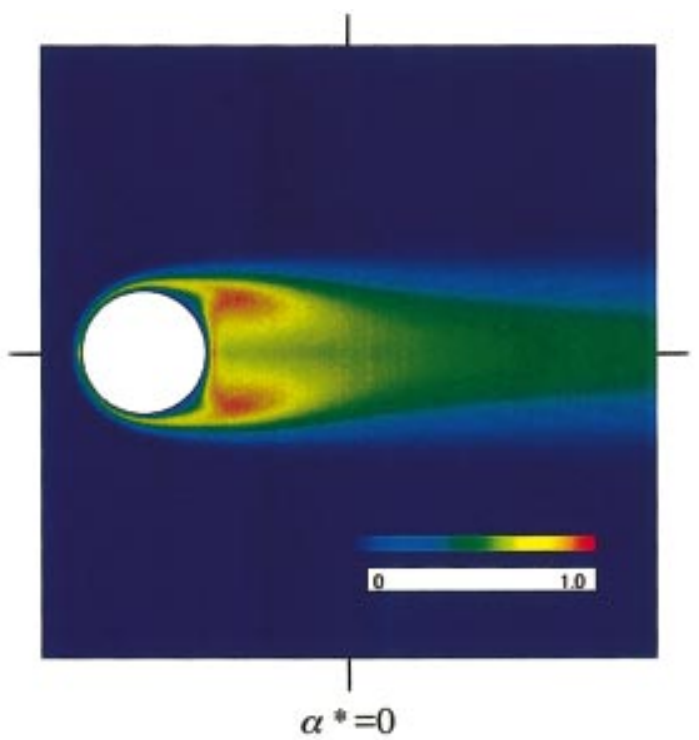

(b)

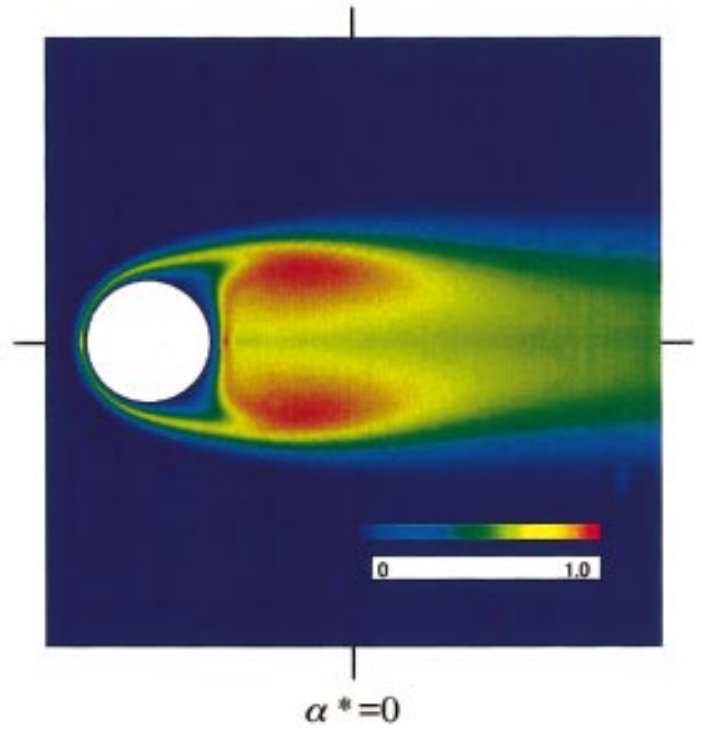

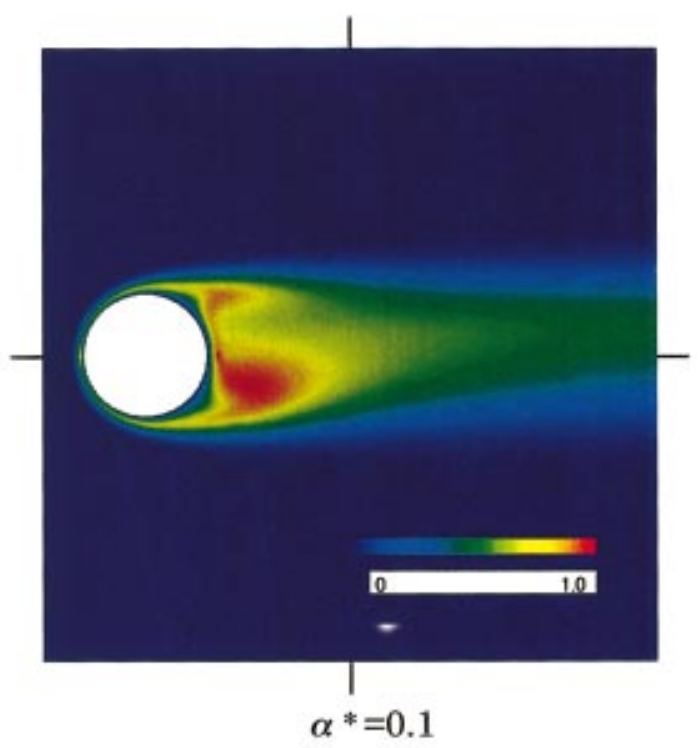

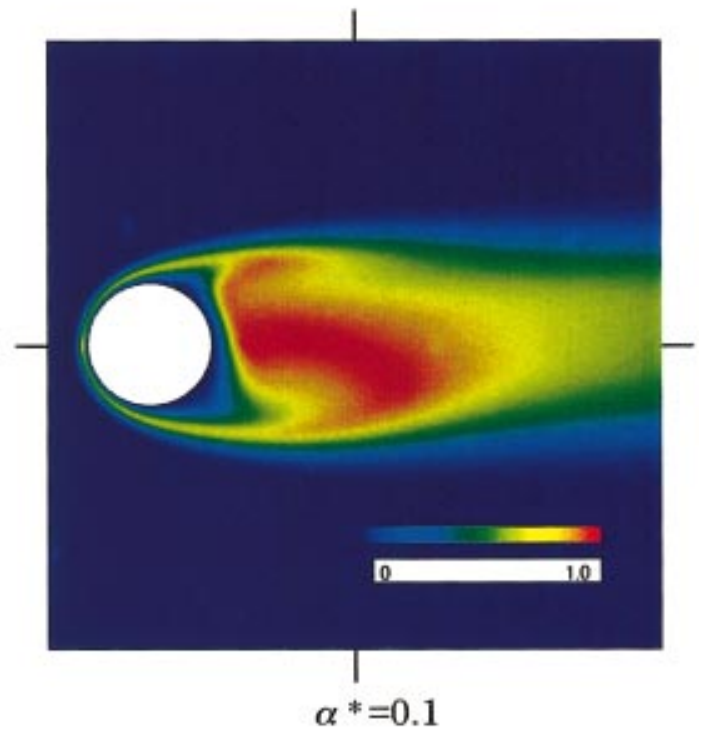

FIG. 15. (Color) Effects of fluid shear rate, $\alpha^{*}$, and outflow velocity, $V_{n}$, on a distribution of product concentration, $C_{P}(\operatorname{Re}=200, \mathrm{Da}=0.1):\left(\right.$ a) $V_{n}=0$; (b) $V_{n}=0.1$.

number, $\mathrm{Sh}(\mathrm{Re}=200)$. As generally known, $\mathrm{Sh}$ for $V_{n}=0$ increases with increasing $\mathrm{Re}$ from the asymptotic value of 2.0 for the $\mathrm{Re} \rightarrow 0$ limit. Also, these figures indicate that $\mathrm{Sh}$ is affected by $V_{n}$ and $\mathrm{Da}$, but not by $\alpha^{*}$. Sh decreases with increasing $V_{n}$ and the discrepancy between $V_{n}=0$ and $V_{n}$ $=0.1$ enlarges as Re increases, whereas $\mathrm{Sh}$ for $\mathrm{Da}=0.1$ increases from the value of $\mathrm{Da}=0$ and the increasing rate seems to be independent of Re. Regarding the effect of the fluid shear, Dandy and Dwyer, ${ }^{13}$ who performed a threedimensional numerical simulation of a linear shear flow around a heated solid sphere, obtained the similar result to ours. They stated that $\mathrm{Nu}$ averaged on the surface of the solid sphere is essentially independent of the fluid shear rate. However, Misumi et al. ${ }^{20}$ and Komori and Misumi ${ }^{21}$ recently did a similar numerical simulation of a linear shear flow around a bubble with a slip surface boundary, and showed that although Sh averaged on the bubble is not affected by the fluid shear either, the values of Sh on the local places, referred to as the local Sherwood number, $\mathrm{Sh}_{\text {local }}$, are clearly different especially on the front side of the bubble.

To provide the insight into the behavior of the mass transfer rate on the local place, $\mathrm{Sh}_{\text {local }}$, the effect of the fluid shear rate, $\alpha^{*}$, together with the effects of the Reynolds number, Re, the outflow velocity, $V_{n}$, and the Damköhler number, $\mathrm{Da}$ on the $\mathrm{Sh}_{\text {local }}$ distribution at $\theta=0$ against $\phi$ are shown in Fig. 17. Here, the effects of $V_{n}, \alpha^{*}$, and Da are plotted for $\operatorname{Re}=200$. In Fig. 17(c) for the effect of $\alpha^{*}$, the distributions of top $(\theta=0)$ and bottom $(\theta=\pi)$ are shown. It is found that, although $\mathrm{Sh}_{\text {local }}$ on the slip surface is reported to monotonously decrease with $\phi^{20}, \mathrm{Sh}_{\text {local }}$ on the nonslip sur- 
(a)

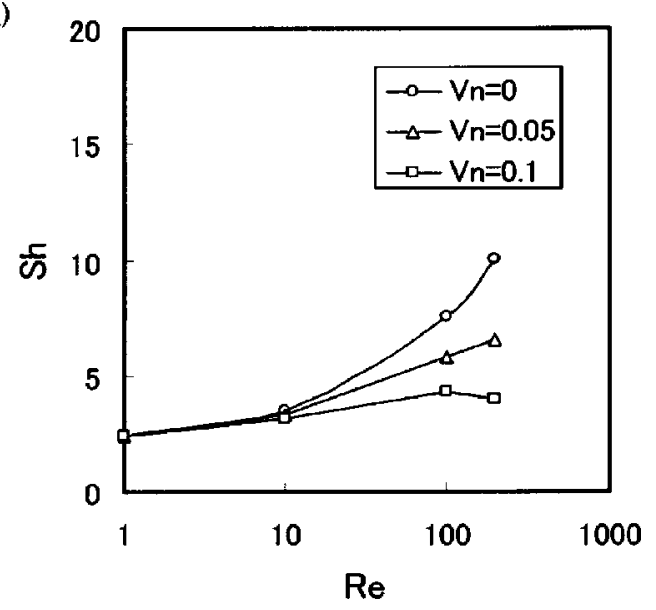

(b)

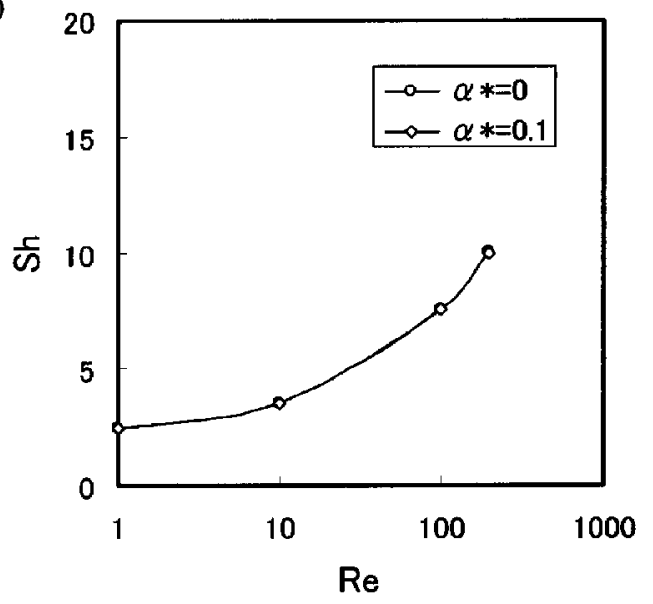

(c)

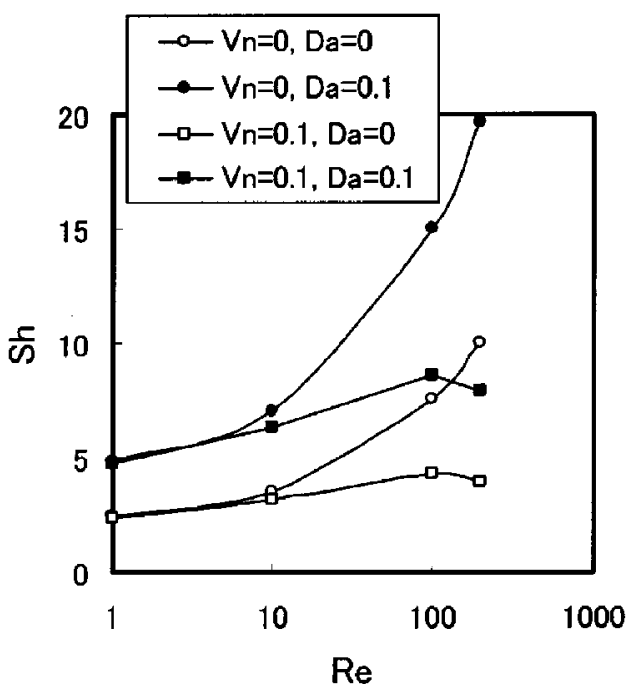

FIG. 16. Effects of outflow velocity, $V_{n}$, fluid shear rate, $\alpha^{*}$, and Damköhler number, Da, on Sherwood number, $\mathrm{Sh}(\mathrm{Re}=200)$ : (a) effect of $V_{n}$; (b) effect of $\alpha^{*}$; (c) effect of Da.

face indicates a minimum value around $\phi=3 \pi / 4$ and increases with $\phi$ again. This variation is considered to be due to the existence of the points of the flow separation and the vortices behind the solid sphere (this will be discussed in detail later). With increasing $\mathrm{Re}, V_{n}$ and $\mathrm{Da}, \mathrm{Sh}_{\text {local }}$ increases, decreases, and increases, respectively, in the whole $\phi$ region. Similarly, despite the coincident of $\mathrm{Sh}$, the $\mathrm{Sh}_{\text {local }}$ distribution for $\alpha^{*}=0.1$ is found to deviate from that for $\alpha^{*}=0$. In the front region of $\phi \leqslant \pi / 2, \mathrm{Sh}_{\text {local }}$ for $\alpha^{*}=0.1$ is greater than that for $\alpha^{*}=0$ on the top and less on the bottom. This trend agrees well with that of Misumi et al. ${ }^{20}$ and Komori and Misumi. ${ }^{21}$ However, unlike their result, ${ }^{20}$ the difference in $\mathrm{Sh}_{\text {local }}$ between that on the top and the bottom is seen also in the rear region of $3 \pi / 4 \leqslant \phi$, and this is rather prominent.

Above $\mathrm{Sh}_{\text {local }}$ behaviors are considered to be strongly related to the tangential viscous stress on the surface of the sphere because the increase in the tangential viscous stress enlarges the scalar gradient, which is proportional to $\mathrm{Sh}_{\text {local }}$. The minimum value of $\mathrm{Sh}_{\text {local }}$ around $\phi=3 \pi / 4$ and the increase in $\mathrm{Sh}_{\text {local }}$ in the rear region of $3 \pi / 4 \leqslant \phi$ is caused by the facts that the tangential viscous stress around $\phi=3 \pi / 4$ indicates the low value, because this location corresponds to the point of the flow separation and that the vortex generated behind the solid sphere magnifies the tangential viscous stress on the rear side. Also, with increasing Re and $V_{n}$, the tangential viscous stress on the surface of the sphere increases and decreases, therefore $\mathrm{Sh}_{\text {local }}$ increases and decreases in the whole $\phi$ region, respectively [Figs. 17(a), 17(b)]. Furthermore, the effect of the fluid shear on the behavior of $\mathrm{Sh}_{\text {local }}$ can be explained as follows [Fig. 17(c)]. In the linear shear flow, the tangential viscous stress on the front side is greater on the top than that on the bottom because of the velocity difference, which makes $\mathrm{Sh}_{\text {local }}$ greater on the top. On the rear side, the tangential viscous stress becomes stronger on the top than that on the bottom due to the influent from the lower side to the upper side (arrow $A$ in Fig. 9). In addition, compared to $\mathrm{Sh}_{\text {local }}$ for $\alpha^{*}=0, \phi$ showing the minimum $\mathrm{Sh}_{\text {local }}$ for $\alpha^{*}=0.1$ are observed to slightly shift upstream on the top but downstream on the bottom, and this trend is similar to that of the points of flow separation shown in Fig. 9. This also suggests the close relationship between $\mathrm{Sh}_{\text {local }}$ and the tangential viscous stress. The reason why $\mathrm{Sh}_{\text {local }}$ increases for $\mathrm{Da}=0.1$ is that the scalar consumption due to the reaction makes the scalar gradient enlarge [Fig. 17(d)].

\section{CONCLUSIONS}

A three-dimensional numerical simulation of an isothermal flow past a solid sphere with outflow in a linear shear flow was performed to investigate the effects of the outflow on the drag and the shear lift. In addition, the effects of the outflow and the fluid shear on the diffusion and reaction of reactant from the surface of the sphere were also discussed. The main results from this study can be summarized as follows.

The outflow from the surface of the sphere reduces the friction drag, increases the pressure drag, and reduces the total drag since the rate of reduction of the friction drag is greater than that of increase of the pressure drag. Also, the rate of change increases with an increase of the particle Reynolds number, and this effect tends to be overestimated with the generally used empirical expression, especially for the 

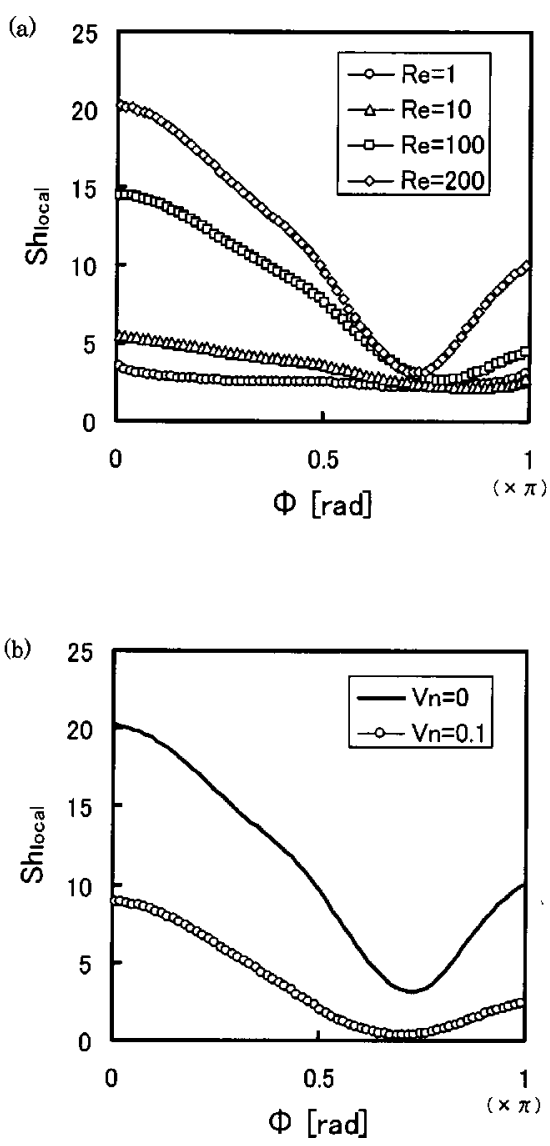

(c)

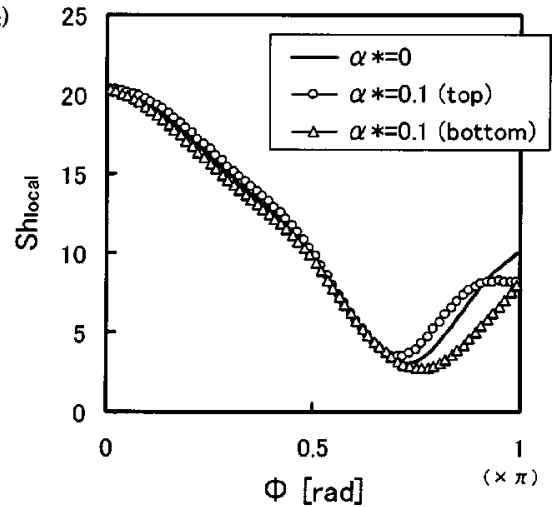

(d)

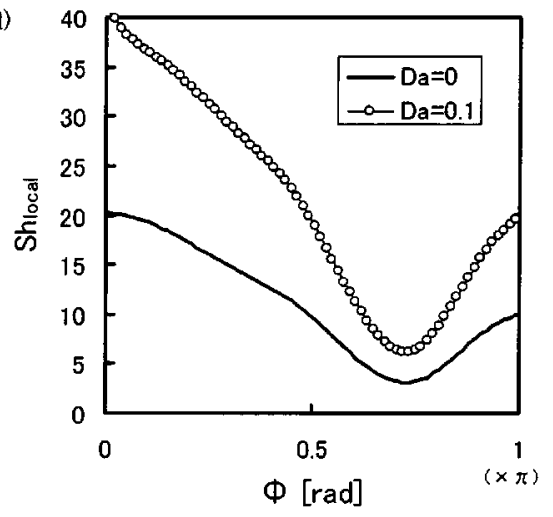

FIG. 17. Effects of Reynolds number, Re, outflow velocity, $V_{n}$, fluid shear rate, $\alpha^{*}$, and Damköhler number, Da, on local Sherwood number, $\mathrm{Sh}_{\text {local }}(\mathrm{Re}$ $=200$ ): (a) effect of Re; (b) effect of $V_{n}$; (c) effect of $\alpha^{*}$; (d) effect of Da. larger particle Reynolds numbers. On the other hand, in the linear shear flow, the outflow acts to push the sphere to the lower fluid velocity side and to promote the negative lift for the high particle Reynolds numbers. This behavior is mainly attributed to the friction lift for the low particle Reynolds numbers, but to the pressure lift for the high particle Reynolds numbers. The diffusion and reaction of reactant from the surface of the sphere are strongly affected by the outflow and the fluid shear because of the deformation of the vortices appeared behind the sphere. This suggests that it is of great importance to take these effects into account in precisely predicting the scalar concentrations and their reactions in the fields, including the evaporating and reacting particles.

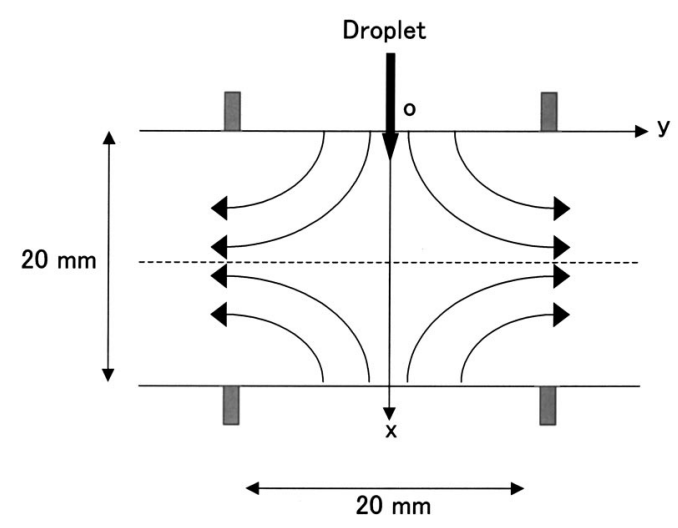

FIG. 18. Schematic of computational domain of planar two-dimensional counterflow field.

\section{ACKNOWLEDGMENTS}

The authors would like to thank Dr. R. Misumi of Yokosuka National University for many helpful discussions. The third author (S.K.) would like to acknowledge the financial support by the Japanese Ministry of Education, Science, and Culture through Grants-in-Aid (No. 14102016).

\section{APPENDIX: ESTIMATION OF OUTFLOW VELOCITY}

In this appendix, typical outflow velocity is estimated by performing a two-dimensional numerical simulation of

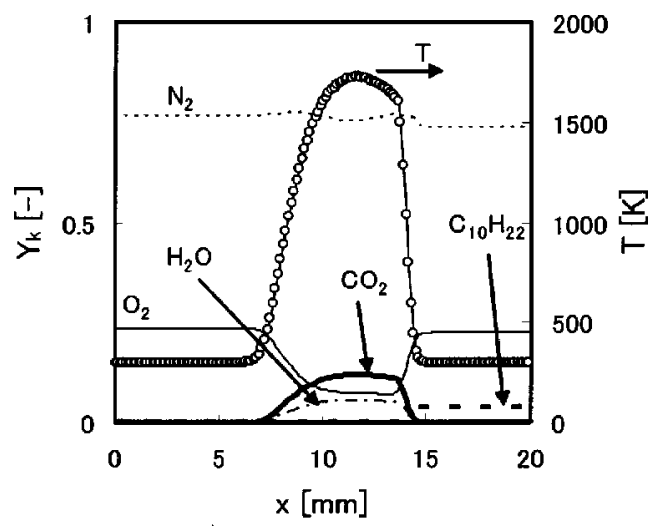

FIG. 19. Profiles of mass fractions of gaseous species, $Y_{k}$, and gas temperature, $T$, with an $x$ axis before droplet injection. 

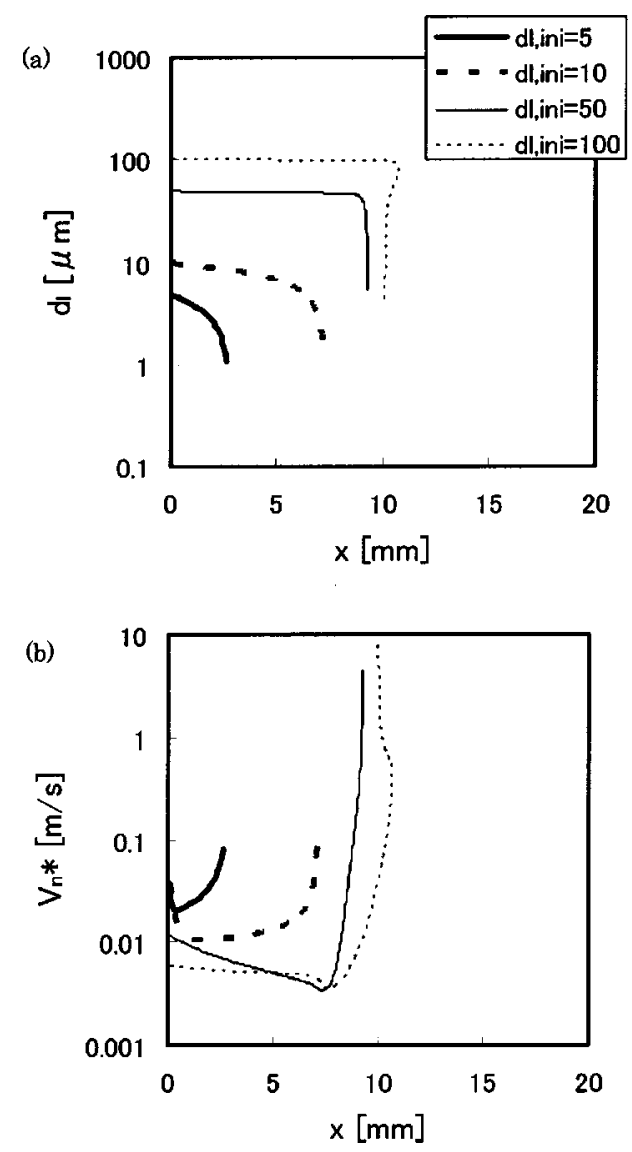

FIG. 20. Variations of droplet diameter, $d_{l}$, and outflow velocity from the droplet surface, $V_{n}^{*}$; (a) droplet diameter, $d_{l}$; (b) outflow velocity, $V_{n}^{*}$.

single droplet combustion in a laminar counterflow. The numerical procedure used is essentially the same as that in Akamatsu et al. ${ }^{28,29}$

The planar two-dimensional numerical flow field for estimating the typical outflow velocity due to the evaporation is shown in Fig. 18. The geometry of the burner port is 20 $\mathrm{mm}$ in width and the port separation is $20 \mathrm{~mm}$. The origin of the calculation domain is located at the center of the upper rectangular burner port, from which a droplet of $n$-decane $\left(\mathrm{C}_{10} \mathrm{H}_{22}\right)$ is supplied. The calculation domain $(0 \mathrm{~mm} \leqslant x$ $\leqslant 20 \mathrm{~mm},-10 \mathrm{~mm} \leqslant y<10 \mathrm{~mm}$ ) is divided into $157 \times 153$ equally spaced computational grids in $x$ and $y$ directions, respectively, which generates the actual control volume size of $130 \mu \mathrm{m} \times 130 \mu \mathrm{m}$. The calculation time step is set at $0.1875 \mathrm{~ms}$ initially, and at $0.375 \mu$ s when $420 \mathrm{~T}>K$ or $d_{1}$ $<20 \mu \mathrm{m}$. From the upper port, atmospheric air $(T=300 \mathrm{~K}$, $P=0.1013 \mathrm{MPa}$, and oxygen mass fraction $\left.Y_{\mathrm{O}_{2}}=0.2357\right)$ is issued at the velocity of $0.4 \mathrm{~m} / \mathrm{s}$. From the lower port, the premixture of atmospheric air and $n$-decane vapor (equivalence ratio, $\left.\phi_{g}=0.6\right)$ is issued in the region of $-3 \mathrm{~mm} \leqslant y$ $\leqslant 3 \mathrm{~mm}$, and atmospheric air is issued in the region of $-10 \mathrm{~mm} \leqslant y<-3 \mathrm{~mm}$ and $3 \mathrm{~mm}<y \leqslant 10 \mathrm{~mm}$, at the velocity of $0.4 \mathrm{~m} / \mathrm{s}$. The gaseous flame is stabilized in a laminar counterflow, stretch ratio of which is $401 / \mathrm{s}$. At a moment, $t=0 \mathrm{~ms}$, an $n$-decane droplet is injected from the center of the upper port at the velocity of $0.4 \mathrm{~m} / \mathrm{s}$.

Gaseous species considered in the calculations are $\mathrm{O}_{2}$,
$\mathrm{N}_{2}, \mathrm{CO}_{2}, \mathrm{H}_{2} \mathrm{O}$, and $\mathrm{C}_{10} \mathrm{H}_{22}$, and their transport properties and thermodynamic data are obtained from CHEMKIN. ${ }^{30,31}$ Properties of liquid $n$-decane are obtained from Abramzon and Siringnano. ${ }^{32}$ The governing equations considered for the gaseous phase (mass, momentum, energy, and species mass) are discretized and solved by the finite volume method using the SIMPLE algorithm. ${ }^{33}$ A single droplet is tracked in a Lagrangian manner. Mass, heat, and momentum interchanges between the gaseous and the single droplet are calculated by the method of the PSI-Cell model. ${ }^{34}$ Regarding the evaporation of droplet, a spherically symmetric, unsteady droplet model with variable properties proposed by Abramzon and Sirignano ${ }^{32}$ is employed.

The computations were performed for the $n$-decane $\left(\mathrm{C}_{10} \mathrm{H}_{22}\right)$ droplets with four different initial diameters of $d_{l, \text { ini }}=5,10,50$ and $100 \mu \mathrm{m}$. Simulations were run 1000 time steps before the stable flame was achieved and 200 time steps after the droplet was injected.

Figure 19 shows the profiles of the mass fractions of the gaseous species of $\mathrm{O}_{2}, \mathrm{~N}_{2}, \mathrm{CO}_{2}, \mathrm{H}_{2} \mathrm{O}$, and $\mathrm{C}_{10} \mathrm{H}_{22}, Y_{k}$, and the gas temperature, $T$, with the $x$ axis before the single $\mathrm{C}_{10} \mathrm{H}_{22}$ droplet is injected. The high $T$ zone appeared in the region of $x=7-15 \mathrm{~mm}$ in the laminar counterflow, and, in this high-temperature zone, $\mathrm{C}_{10} \mathrm{H}_{22}$ vapor supplied from the lower port of $x=20 \mathrm{~mm}$ rapidly disappears, $\mathrm{O}_{2}$ consumes, and the $\mathrm{CO}_{2}$ and $\mathrm{H}_{2} \mathrm{O}$ form.

The variations of the droplet diameter, $d_{l}$, and the outflow velocity, $V_{n}^{*}$, for the single $\mathrm{C}_{10} \mathrm{H}_{22}$ droplet with initial diameters of $d_{l, \text { ini }}=5,10,50$ or $100 \mu \mathrm{m}$ are shown in Fig. 20. It is found that the smaller droplets with $d_{l \text {,ini }}=5$ and 10 $\mu \mathrm{m}$ disappears before arriving at the high-temperature zone, whereas the larger droplets with $d_{l, \text { ini }}=50$ and $100 \mu \mathrm{m}$ get into the high temperature zone and instantaneously evaporate. Accordingly, $V_{n}^{*}$ of the smaller droplets with $d_{l, \text { ini }}=5$ and $10 \mu \mathrm{m}$ gradually increase as the droplets approach the high-temperature zone, and those of the larger droplets with $d_{l, \text { ini }}=50$ and $100 \mu \mathrm{m}$ tend to keep the low values before the high-temperature zone and rapidly increase in it. What is particularly noteworthy is that the magnitudes of $V_{n}^{*}$ are quite different between the droplets that nearly disappear before the high-temperature zone and those that penetrate and mainly evaporate in this zone. Indeed, $V_{n}^{*}$ for the latter droplets is about two orders of magnitude larger than that for the former ones. The maximum $V_{n}^{*}$ for the droplets evaporating outside and inside the high-temperature zone can be evaluated to be of $10^{-2}$ and $10^{0}$ orders of magnitudes $(\mathrm{m} / \mathrm{s})$, respectively.

${ }^{1}$ L. Schiller and J. Lumley, "On the equation of motion for a particle in turbulent fluid," Appl. Sci. Res., Sect. A 6, 114 (1956).

${ }^{2}$ S. A. Morsi and A. J. Alexander, "An investigation of particle trajectories in two-phase systems," J. Fluid Mech. 55, 193 (1972).

${ }^{3}$ G. H. Bailey, I. W. Slater, and P. Eisenklam, "Dynamic equations and solutions for particles undergoing mass transfer," Br. Chem. Eng. 15, 912 (1970).

${ }^{4}$ P. Chuchottaworn, A. Fujinami, and K. Asano, "Numerical analysis of the effect of mass injection or suction on drag coefficients of a sphere," J. Chem. Eng. Jpn. 16, 18 (1982).

${ }^{5}$ M. Renksizbulut and M. C. Yuen, "Experimental study of droplet evaporation in a high-temperature air stream," ASME J. Heat Transfer 105, 384 (1983). 
${ }^{6}$ K. Asano, I. Taniguchi, K. Maeda, and H. Kosuge, "Simultaneous measurement of drag coefficients and mass transfer of a volatile drop falling freely,” J. Chem. Eng. Japan 21, 367 (1988).

${ }^{7}$ M. Renksizbulut and M. C. Yuen, "Numerical study of droplet evaporation in a high-temperature stream," ASME J. Heat Transfer 105, 389 (1983).

${ }^{8}$ R. I. Sujith, G. A. Waldherr, J. I. Jagoda, and B. T. Zinn, "On the effect of evaporation on droplet drag," ASME J. Fluids Eng. 118, 863 (1996).

${ }^{9}$ P. G. Saffman, "The lift on a small sphere in a shear flow," J. Fluid Mech. 22, 385 (1965); corrigendum, ibid. 31, 624 (1968).

${ }^{10} \mathrm{~J}$. B. McLaughlin, "Inertial migration of a small sphere in linear shear flow," J. Fluid Mech. 224, 261 (1991).

${ }^{11} \mathrm{R}$. Mei, "An approximate expression for the shear lift force on a spherical particle at finite Reynolds number," Int. J. Multiphase Flow 18, 145 (1992).

${ }^{12} \mathrm{R}$. Kurose and S. Komori, "Drag and lift forces on a rotating sphere in a linear shear flow," J. Fluid Mech. 384, 183 (1999).

${ }^{13}$ D. Dandy and H. Dwyer, "A sphere in shear flow at finite Reynolds number: effect of shear on particle lift, drag, and heat transfer," J. Fluid Mech. 216, 381 (1990).

${ }^{14} \mathrm{~S}$. M. Tieng and A. C. Yan, "Experimental investigation on convective heat transfer of heated spinning sphere," Int. J. Heat Mass Transfer 36, 599 (1993)

${ }^{15}$ H. D. Nguyen, S. Paik, and J. Chung, "Unsteady mixed convection heat transfer from a solid sphere: the conjugate problem," Int. J. Heat Mass Transfer 36, 4443 (1993).

${ }^{16} \mathrm{H}$. Chattopadhyay and S. K. Dash, "Numerical visualization of convective heat transfer from a sphere-with and without radial mass efflux," Int. J. Numer. Methods Heat Fluid Flow 5, 705 (1995).

${ }^{17}$ N. Kawahara, A. L. Yarin, G. Brenn, O. Kastner, and F. Durst, "Effect of acoustic streaming on the mass transfer from a sublimating sphere," Phys. Fluids 12, 912 (2000).

${ }^{18} \mathrm{~S}$. Balachander and M. Y. Ha, "Unsteady heat transfer from a sphere in a uniform cross-flow," Phys. Fluids 13, 3714 (2001).

${ }^{19} \mathrm{H}$. M. Blackburn, "Mass and momentum transport from a sphere in steady and oscillatory flows," Phys. Fluids 14, 3997 (2002).

${ }^{20} \mathrm{R}$. Misumi, M. Ishidu, and S. Komori, "The effects of bubbles on mass transfer across breaking air-water interface," Trans. Jpn. Soc. Mech. Eng., Ser. B 66, 2327 (2000) (in Japanese).

${ }^{21} \mathrm{~S}$. Komori and R. Misumi, "The effects of bubbles on mass transfer across the breaking air-water interface," in Gas Transfer at Water Surfaces, AGU Geophysical Monograph Series 127, edited by M. A. Donelan, W.
M. Drennan, E. S. Saltzman, and R. W. Wanninkof (American Geophysical Union, Washington, D.C., 2002), pp. 285-290.

${ }^{22} \mathrm{H}$. Hanazaki, "A numerical study of three-dimensional stratified flow past a sphere," J. Fluid Mech. 192, 393 (1988).

${ }^{23}$ F. C. Thames, J. F. Thompson, C. W. Mastin, and R. L. Walker, "Numerical solutions for viscous and potential flow about arbitrary twodimensional bodies using body-fitted coordinate systems," J. Comput. Phys. 24, 245 (1977).

${ }^{24}$ T. Kawahara and K. Kuwahara, "Computation of high Reynolds number flow around a circular cylinder with surface roughness," AIAA Paper 840340, 1987.

${ }^{25} \mathrm{H}$. Schlichting, Boundary-Layer Theory, 6th ed. (McGraw-Hill, New York, 1968)

${ }^{26}$ K. A. Cliffe and D. A. Lever, "Isothermal flow past a blowing sphere," Int. J. Numer. Methods Fluids 5, 709 (1985).

${ }^{27}$ R. S. Miller and J. Bellan, "Direct numerical simulation of a confined three-dimensional gas mixing layer with one evaporating hydrocarbondroplet-laden stream," J. Fluid Mech. 384, 293 (1999).

${ }^{28}$ F. Akamatsu, H. Saitoh, and M. Katsuki, "Numerical study on combustion characteristics of splay flat flames," Proceedings of the 4th KSME/JSME Thermal Engineering Conference 1, 2000, pp. 259-264.

${ }^{29}$ F. Akamatsu, H. Saitoh, and M. Katsuki, "Numerical simulation of splay flat flames stabilized in a laminar counterflow," Proceedings of the 8th Conference on Liquid Atomization and Splay Systems, ICLASS-2000, 2000, pp. 25-32.

${ }^{30}$ R. J. Kee, F. M. Rupley, and J. A. Miller, "Chemkin-II: A Fortran chemical kinetics package for the analysis of gas phase chemical kinetics," SANDIA Report SAND89-8009B, 1989.

${ }^{31}$ R. J. Kee, G. Dixon-Lewis, J. Warnatz, M. E. Coltrin, and J. A. Miller, “A Fortran computer code package for the evaluation of gas-phase multicomponent transport properties," SANDIA Report, SAND86-8246, 1986.

${ }^{32}$ B. Abramzon and W. A. Sirignano, "Approximation theory of a single droplet vaporization in a convective field: effects of variable properties, Stefan flow and transient liquid heating," ASME-JSME Thermal Engineering Joint Conference, 1987, Vol. 1, p. 11.

${ }^{33}$ S. V. Patankar, Numerical Heat Transfer and Fluid Flow (McGraw-Hill, New York, 1980).

${ }^{34}$ C. T. Crowe, M. P. Sharma, and D. E. Stock, "The particle-source-in cell (PSI-CELL) model for gas-droplet flows,” ASME J. Fluids Eng. 99, 325 (1977). 